\title{
Histone acetyltransferase 1 promotes gemcitabine resistance by regulating the PVT1/EZH2 complex in pancreatic cancer
}

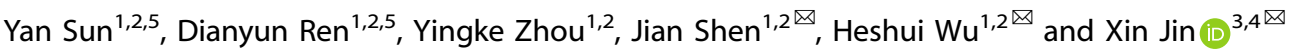

(c) The Author(s) 2021

The poor prognosis of pancreatic cancer is primarily due to the development of resistance to therapies, including gemcitabine. The long noncoding RNA PVT1 (IncRNA PVT1) has been shown to interact with enhancer of zeste 2 polycomb repressive complex 2 subunit (EZH2), promoting gemcitabine resistance in pancreatic cancer. In this study, we found histone acetyltransferase 1 (HAT1) enhanced the tolerance of pancreatic cancer cells to gemcitabine and HAT1-mediated resistance mechanisms were regulated by PVT1 and EZH2. Our results showed that the aberrant HAT1 expression promoted gemcitabine resistance, while silencing HAT1 restored gemcitabine sensitivity. Moreover, HAT1 depletion caused a notable increase of gemcitabine sensitivity in gemcitabineresistant pancreatic cancer cell lines. Further research found that HAT1 increased PVT1 expression to induce gemcitabine resistance, which enhanced the binding of bromodomain containing 4 (BRD4) to the PVT1 promoter, thereby promoting PVT1 transcription. Besides, HAT1 prevented EZH2 degradation by interfering with ubiquitin protein ligase E3 component n-recognin 4 (UBR4) binding to the N-terminal domain of EZH2, thus maintaining EZH2 protein stability to elevate the level of EZH2 protein, which also promoted HAT1-mediated gemcitabine resistance. These results suggested that HAT1 induced gemcitabine resistance of pancreatic cancer cells through regulating PVT1/EZH2 complex. Given this, Chitosan (CS)-tripolyphosphate (TPP)-siHAT1 nanoparticles were developed to block HAT1 expression and improve the antitumor effect of gemcitabine. The results showed that CS-TPP-siHAT1 nanoparticles augmented the antitumor effects of gemcitabine in vitro and in vivo. In conclusion, HAT1-targeted therapy can improve observably gemcitabine sensitivity of pancreatic cancer cells. HAT1 is a promising therapeutic target for pancreatic cancer.

Cell Death and Disease (2021)12:878; https://doi.org/10.1038/s41419-021-04118-4

\section{INTRODUCTION}

Pancreatic cancer is a particularly aggressive and lethal malignancy of the digestive system [1, 2]. Early diagnosis of pancreatic carcinoma is challenging due to its anatomical position; hence, only $15 \%$ of pancreatic cancer patients undergo surgical resection $[3,4]$. Pancreatic cancer has a poor prognosis and extremely high mortality and morbidity rates, with a 5-year survival rate of less than $5 \%[5,6]$.

Chemotherapy with gemcitabine is the treatment of choice for pancreatic cancer patients who were not eligible for surgery [7]. Gemcitabine inhibits pancreatic cancer cell proliferation by replacing cytidine during DNA replication and blocking the biosynthesis of deoxyribonucleotides [8]. However, the development of resistance to gemcitabine is not uncommon among pancreatic cancer patients undergoing treatment [9]. Drug resistance can be internal (innate resistance) or acquired (acquired resistance) after multiple treatment cycles [10]. Findings from large-scale technologies, including proteomics and nextgeneration RNA sequencing, suggest that numerous proteins mediate gemcitabine resistance [11]. For instance, aberrant expression of enhancer of zeste homolog $2(\mathrm{EZH} 2)$ in pancreatic cancer cells has been linked to gemcitabine resistance, possibly due to the downregulation of the tumor suppressor p2 $7^{\text {Kip } 1}$ [12]. Additionally, silencing of the long noncoding RNA (IncRNA) PVT1 increased gemcitabine sensitivity in pancreatic cancer cells $[13,14]$. Interestingly, PVT1 has been found to form a complex with EZH2, a key step in the development of gemcitabine resistance in pancreatic cancer [14].

We have previously shown that aberrant expression of histone acetyltransferase 1 (HAT1) enhanced PD-L1 expression and promoted pancreatic cancer cell proliferation by modulating the function of BRD4. Herein, we show that HAT1 knockdown in pancreatic cancer cells increases gemcitabine sensitivity and decreases PVT1/EZH2 complex levels, suggesting that HAT1 may represent a promising therapeutic target in pancreatic cancer.

\section{RESULTS}

Aberrant HAT1 expression promotes gemcitabine resistance in pancreatic cancer cells

HAT1 is often upregulated in pancreatic cancer and promotes pancreatic cancer cell proliferation by regulating PD-L1

\footnotetext{
${ }^{1}$ Department of Pancreatic Surgery, Union Hospital, Tongji Medical College, Huazhong University of Science and Technology, 430022 Wuhan, China. ${ }^{2}$ Sino-German Laboratory of

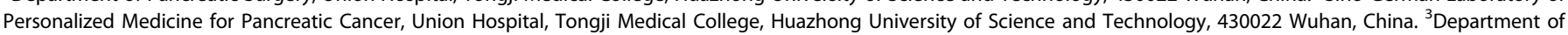

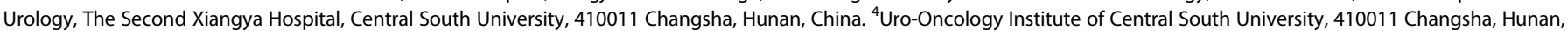
China. ${ }^{6}$ These authors contributed equally: Yan Sun, Dianyun Ren. ${ }^{凶}$ email: 1024263571@qq.com; heshuiwu@hust.edu.cn; jinxinxy2@csu.edu.cn Edited by Professor Anastasis Stephanou
}

Received: 25 January 2021 Revised: 18 May 2021 Accepted: 19 May 2021

Published online: 25 September 2021 
expression; however, the oncogenic role of HAT1 in pancreatic cancer remains poorly understood. Several studies had found that the expression of histone acetyltransferase (HAT) genes was related to drug resistance [15-17]. As is well known, pancreatic cancer was easily tolerated by chemotherapy, while upregulated HAT1 might led to the results. Thus, we explored the influence of abnormal HAT1 expression on the sensitivity of several commonly used drugs in pancreatic cancer cells, which showed the significantly reduced IC50 for gemcitabine when HAT1 was knocked down, while the sensitivity of other drugs was slightly reduced or unchanged (Fig. 1a). Furtherly, HAT1 silencing increased three pancreatic cancer cell lines sensitivity to gemcitabine; HAT1 overexpression had the opposite effect (Fig. $1 \mathrm{~b}$ and Fig. S1a, b). The expression of HAT1 also was upregulated obviously in pancreatic cancer cells compared to the normal cells (Fig. S1c), which might be the potential cause of gemcitabine resistance. Additionally, MTS and colony formation assays revealed that HAT1-knockdown cells grew slower than control cells in the presence of gemcitabine (Fig. 1c, d and Fig. S1d, e). Moreover, HAT1 silencing in pancreatic cancer cells enhanced apoptosis in response to gemcitabine treatment (Fig. 1e, $f$ and Fig. S1f). Consistently, HAT1 knockdown enhanced the tumor-suppressive effects of gemcitabine in tumor xenografts (Fig. 1g). Specifically, tumors with HAT1 knockdown exhibited the slowest tumor growth, the highest caspase-3 levels, and the lowest Ki67 levels in response to gemcitabine treatment (Figs. $1 \mathrm{~h}-\mathrm{j}$ ).

Besides, we constructed the gemcitabine-resistant PANC-1. The cell morphology changed significantly and the sensitivity reduced by 7.79 times compared to ordinary PANC-1 (Fig. S1g and S1h). GR-PANC-1 was also used to carry out MTS, clone formation and caspase-3 activity detecting (Fig. S1i-k). The results showed that silencing HAT1 could recover the gemcitabine sensitivity. Moreover, the expression of HAT1 increased in GR-PANC-1, which further illustrated HAT1 promoted the gemcitabine resistance of pancreatic cancer cells (Fig. S1I). These findings suggest that aberrant HAT1 expression affects the response of pancreatic cancer cells to gemcitabine in vitro and in vivo.

\section{HAT1 enhances PVT1 expression by facilitating BRD4 binding to PVT1 promoter to promote gemcitabine resistance}

Despite evidence of the critical role of HAT1 in pancreatic cancer progression and gemcitabine resistance, the underlying mechanism remains elusive. RNA sequencing of HAT1 knockdown and control PANC-1 cells yielded 945 differentially expressed genes, including 490 upregulated and 455 downregulated genes (Fig. 2a and Fig. S2a). A significant portion of these genes was directly or indirectly related to cancer (Fig. S2b). Importantly, the IncRNA PVT1, a potential downstream target gene of HAT1, was downregulated upon HAT1 silencing, whereas HAT1-overexpressing cells exhibited elevated PVT1 levels (Fig. 2b and Fig. S2b, c). Furthermore, analyses using the GEPIA tool and ENCORI PanCancer Analysis Platform indicated a positive correlation between HAT1 and PVT1 expression levels (Fig. 2c, d), suggesting that HAT1 regulates PVT1 expression in pancreatic cancer cells. A previous genome-wide screen identified PVT1 as a regulator of gemcitabine sensitivity in pancreatic cancer cells [13]. MTS assay confirmed that PVT1 was a drug-related resistant gene (Fig. S2d). To verify the relevance of PVT1 in HAT1-mediated gemcitabine resistance, we performed MTS assay and found that cells with HAT1 silencing and PVT1 overexpressing proliferated significantly faster than HAT1-knockdown cells (Fig. S2e). Thus, HAT1 promotes gemcitabine resistance by enhancing PVT1 expression.

Although our findings demonstrate that HAT1 promotes PVT1 transcription, the underlying molecular mechanism is poorly understood. HAT1 could catalyze H4 acetylation (Fig. S3a, b), which is essential for the binding of the transcription activator BRD4 to histone H4 [18]. Besides, we have previously shown that HAT1 promoted PD-L1 expression in pancreatic cancer cells in a
BRD4-dependent manner. We analyzed available ChIP-seq data of BRD4 [19] and identified a BRD4-binding peak in the promoter of PVT1 (Fig. 2e). This result was confirmed in pancreatic cancer cells by ChIP-qPCR (Fig. 2f). Moreover, PVT1 expression levels were decreased or increased after BRD4 silencing or overexpression, respectively (Fig. $2 \mathrm{~g}$ and Fig. S3c), suggesting that BRD4 may regulate the transcription of PVT1. Furthermore, analysis using GEPIA indicated a strong positive correlation between BRD4 and PVT1 levels in pancreatic cancer specimens (Fig. 2h). To assess the relevance of BRD4 in the HAT1-mediated regulation of PVT1 expression, we silenced BRD4 in combination with HAT1 knockdown or HAT1 overexpression. Interestingly, BRD4 silencing attenuated the ability of HAT1 to regulate PVT1 expression (Fig. 2i). Similarly, treatment with the BRD4 inhibitor JQ1 abrogated the ability of HAT1 silencing or overexpression to regulate PVT1 expression levels (Fig. 2j). The ability of BRD4 to bind to the PVT1 promoter was also significantly reduced upon JQ1 treatment, even in HAT1-overexpressing cells (Fig. 2k). Besides, the therapeutic effects of gemcitabine were significantly enhanced whether using JQ1 to inhibit BRD4 function or directly knocking down BRD4 (Fig. S3d, e), which further indicated that BRD4 could regulate the expression of PVT1. Collectively, these data suggest that the ability of HAT1 to induce PVT1 expression in pancreatic cancer cells requires BRD4 (Fig. 2l).

\section{HAT1 stabilizes EZH2 to promote gemcitabine resistance by competing with UBR4 for binding to the $\mathrm{N}$-terminal domain of EZH2}

Several researches showed that EZH2 was a drug-related resistant gene $[14,20,21]$, our research also confirmed EZH2 made pancreatic cancer cells become insensitive to gemcitabine (Fig. S4a, b). PVT1 had also been shown to bind EZH2 [22, 23], it was consistent in pancreatic cancer cells. PVT1 did not change the mRNA level of EZH2 (Fig. S4c), but could bind to EZH2 protein (Fig. S4d, e). Thus, we tried to detect the correlation between HAT1 and EZH2. First, the appropriate working concentration and duration of GSK126 (10 $\mu \mathrm{M}$ and 3 days) were screened in pancreatic cancer cells (Fig. S4f). We found that EHZ2 inhibition with GSK126 or silence suppressed HAT1-mediated gemcitabine resistance (Fig. $3 a$ and Fig. $54 \mathrm{~g}-\mathrm{i})$, suggesting that $\mathrm{EZH} 2$ is required for the ability of HAT1 to promote gemcitabine resistance. We also found that the protein but not the mRNA levels of EZH2 were decreased after HAT1 silencing in pancreatic cancer cells. Conversely, forced HAT1 expression increased the protein level of $\mathrm{EZH} 2$, although $\mathrm{EZH} 2$ mRNA levels remained unchanged (Fig. 3b, c and Fig. S5a, b). Tissue microarray of pancreatic cancer $(n=31)$ was used to conduct IHC analysis and ascertain the relationship between HAT1 and EZH2 in pancreatic cancer (Fig. S5c). The IHC score of HAT1 and EZH2 was calculated and summarized in a heatmap (Fig. 3d). We observed a positive correlation between the protein levels of HAT1 and EZH2 in pancreatic cancer tissues (Spearman correlation coefficient $r=0.5899, P=0.0005$; Fig. S5d).

Since HAT1 primarily regulated EZH2 expression at the posttranscriptional level, we hypothesized that HAT1 may regulate EZH2 protein stability. Although EZH2 protein levels were reduced after HAT1 silencing, treatment with the proteasome inhibitor MG132 restored EZH2 protein levels in HAT1-knockdown pancreatic cancer cells (Fig. 3e). Additionally, the half-life of EZH2 protein was significantly shorter in pancreatic cells with HAT1 knockdown than in control cells; HAT1 overexpression extended the half-life of EZH2 protein (Fig. 3f). Further, HAT1 silencing increased the polyubiquitination levels of EZH2, and HAT1 overexpression decreased EZH2 polyubiquitination (Fig. $3 g$ ). Coimmunoprecipitation assays revealed an interaction between HAT1 and EZH2 in pancreatic cancer cell lines, regardless of endogenous or exogenous expression (Fig. 3h and Fig. S5e). To identify the EZH2-binding domain of HAT1, we constructed two GST-EZH2 recombinant proteins: a whole-length GST-EZH2 and an 
a

\begin{tabular}{|c|c|c|c|c|c|c|c|c|c|}
\hline 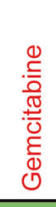 & 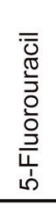 & 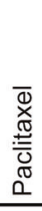 & 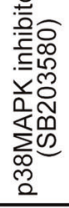 & 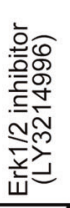 & 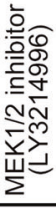 & 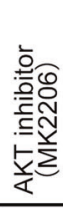 & 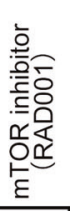 & 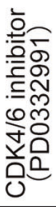 & 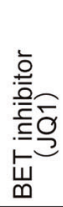 \\
\hline 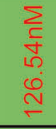 & 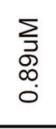 & 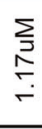 & $\begin{array}{l}\sum_{0} \\
\infty \\
\infty \\
\oplus \\
\underline{0}\end{array}$ & 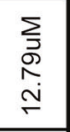 & 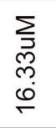 & $\begin{array}{l}\sum \\
\stackrel{\Xi}{8} \\
\stackrel{\leftrightarrow}{\sim}\end{array}$ & 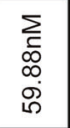 & 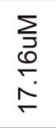 & \\
\hline 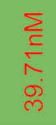 & 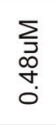 & $\begin{array}{l}\sum \\
\stackrel{\jmath}{\hat{N}} \\
0\end{array}$ & $\begin{array}{l}\sum_{\supset} \\
\substack{+\infty} \\
\stackrel{0}{\sigma}\end{array}$ & $\begin{array}{l}\sum_{0} \\
0 \\
0 \\
0\end{array}$ & 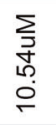 & 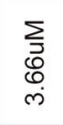 & 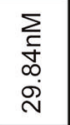 & 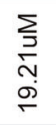 & \\
\hline
\end{tabular}

PANC-1

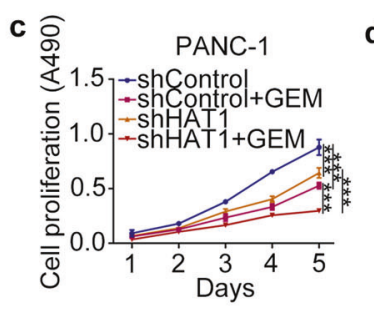

d
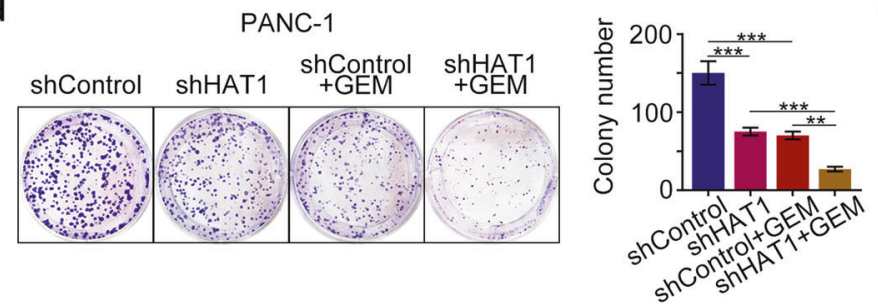

PANC-1
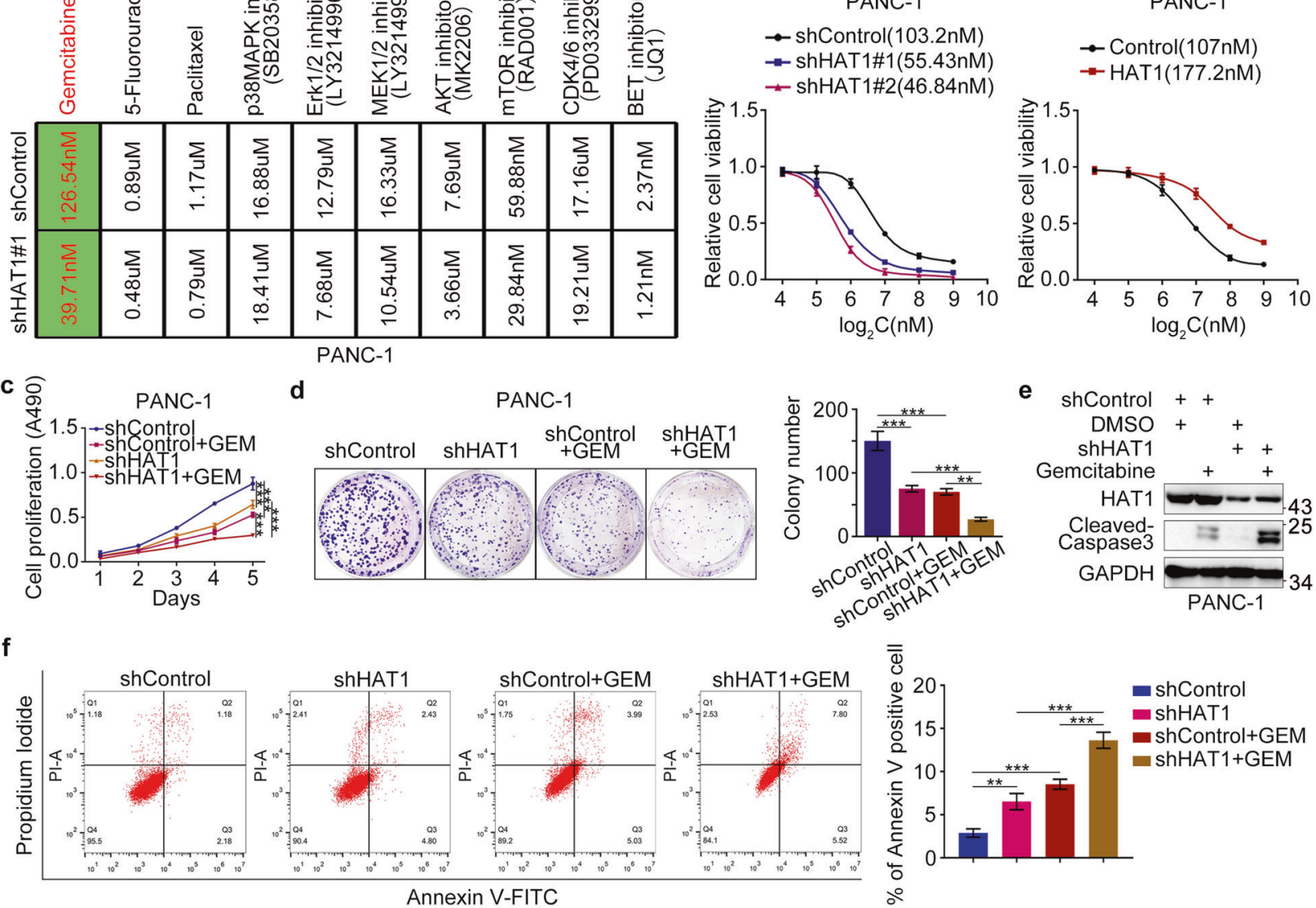

g

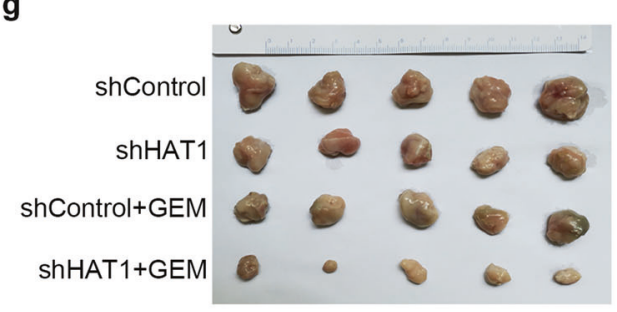

h

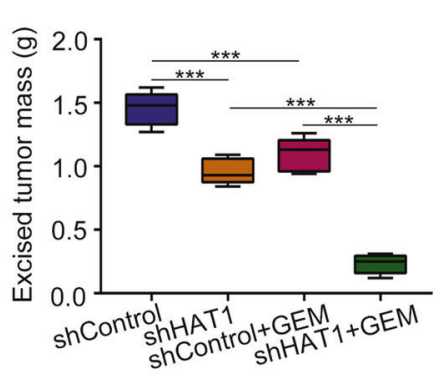

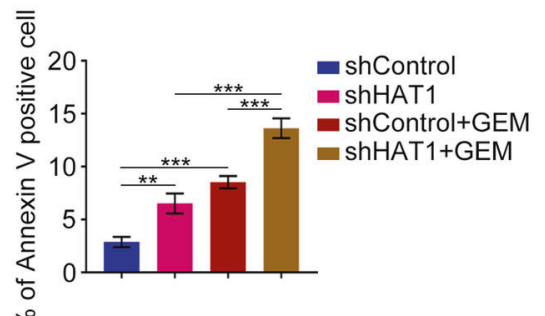

웅

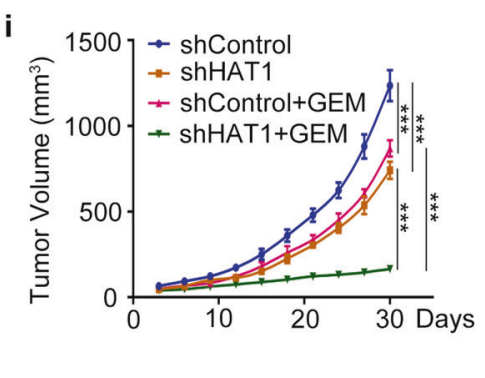

j
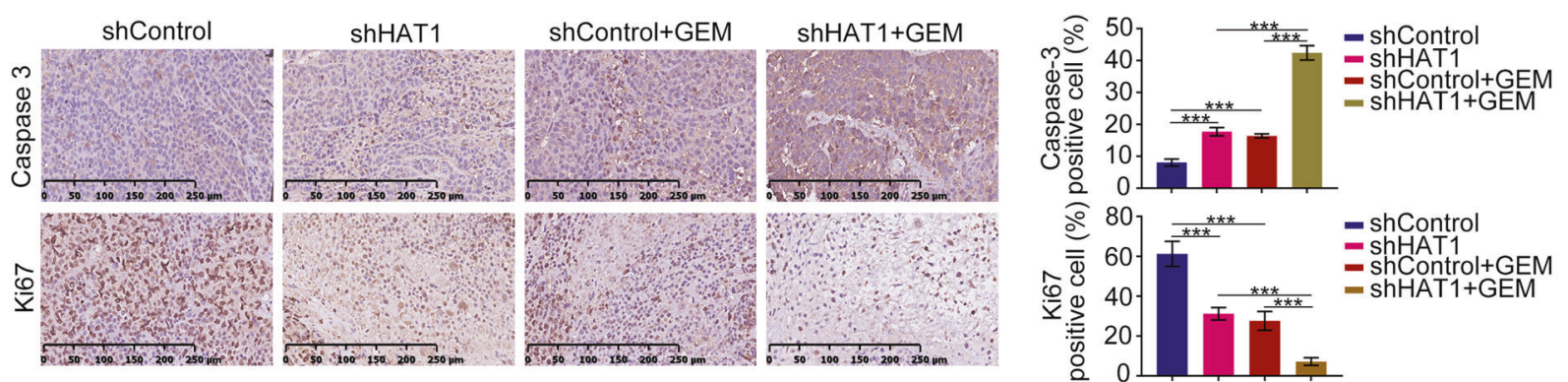

Fig. 1 Aberrant HAT1 expression promotes gemcitabine resistance in pancreatic cancer cells. a MTS assay was used to detect the viability of PANC-1 after treating with different drugs. GraphPad Prism 7.0 software was used to calculate IC50. b MTS assay detected the viability of PANC-1 cells with normal, knockdown, and overexpressing HAT1 after treatment with gemcitabine. The cell viability curve showed IC50 values of gemcitabine among different groups. c-f PANC-1 cells were infected with lentivirus expressing control or HAT1-specific shRNAs. After infecting $72 \mathrm{~h}$, cells were harvested and treated with gemcitabine $(50 \mathrm{nM})$ for MTS assay (c), colony formation assay (d), cleaved caspase-3 activity assay (e), and Annexin-V/Propidium lodide assay (f). All data were shown as mean values \pm SD $(n=3)$. ${ }^{* *} P<0.01 ;{ }^{* * *} P<0.001$. $\mathbf{g}-\mathbf{j}$ PANC-1 cells were transfected with indicated plasmids for $72 \mathrm{~h}$. Cell were subcutanousely injected into the nude mice. These mice were injected intraperitoneally with or without gemcitabine $(10 \mathrm{mg} / \mathrm{kg})$ every 3 days when tumor volume reached to $50 \mathrm{~mm}^{3}$. The tumor volume was measured every 3 days and all tumors were harvested for photograph and weight after 30 days (g-i). All tumors were conducted caspase3 and Ki67 analysis by IHC staining (j). Data presented as Means \pm SD $(n=5)$. ${ }^{* *} P<0.001$. 


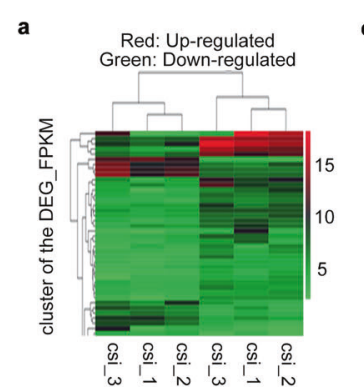

b
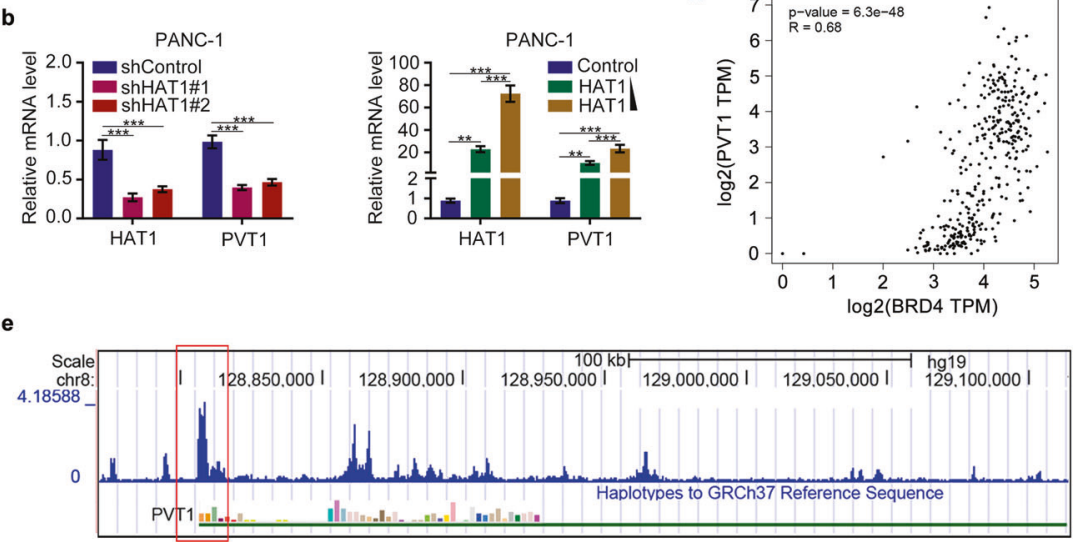

f
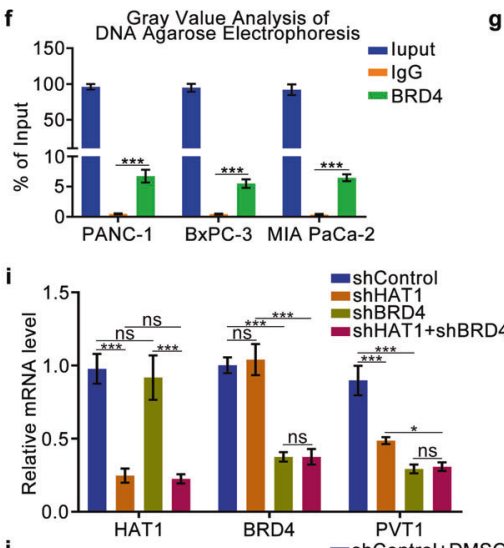

j

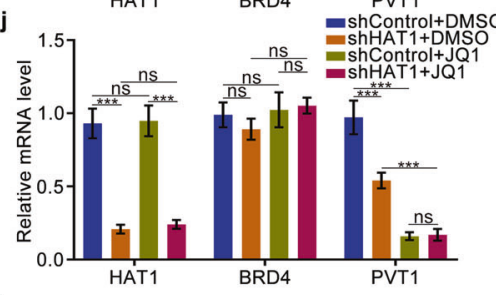

k

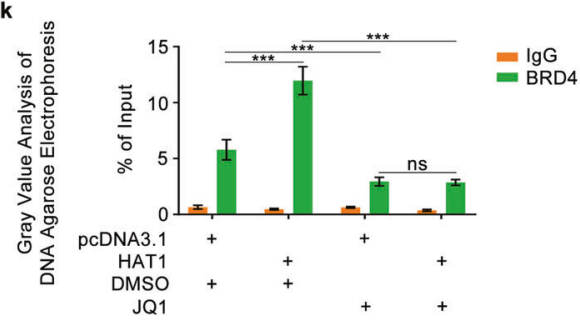

d

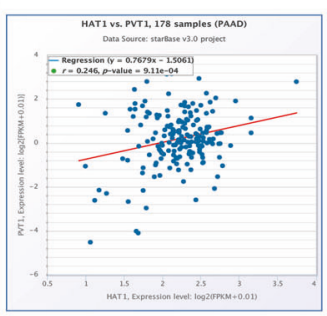

h

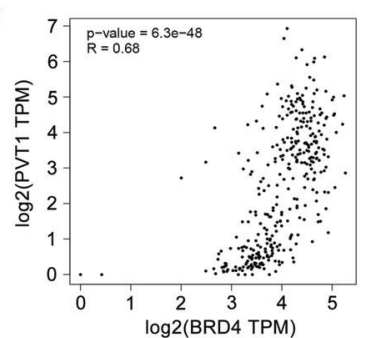

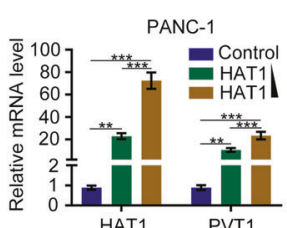
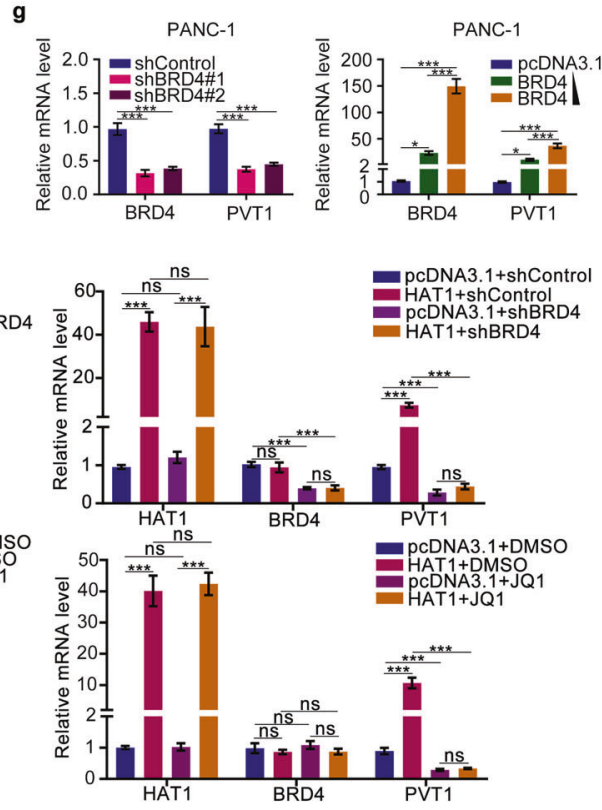

I

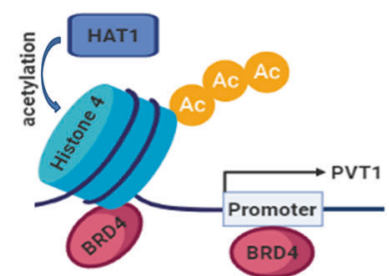

$\mathrm{N}$-terminus-lacking GST-EZH2 (GST- $\Delta \mathrm{N})$ (Fig. 3i). GST pull-down revealed that $\mathrm{EZH} 2$ lacking the $\mathrm{N}$-terminal domain failed to interact with HAT1, indicating that the N-terminal domain of EZH2 is required for its binding to HAT1 (Fig. 3j). The N-terminal WD repeat domain of $\mathrm{EZH} 2$ is believed to be a ubiquitin ligase-binding motif $[24,25]$; as an E3 ubiquitin ligase, UBR4 may bind to the $\mathrm{N}$-terminus of EZH2 to promote EZH2 polyubiquitination [26]. Thus, we hypothesized that HAT1 binds to the N-terminal domain of EZH2, competing with UBR4 binding. Supporting this hypothesis, HAT1 silencing increased the ability of UBR4 to bind EZH2, 
Fig. 2 HAT1 enhances PVT1 expression by facilitating BRD4 binding to PVT1 promoter to promote gemcitabine resistance. a Heatmap showed the differential expressed genes of PANC-1 cells infected by shControl or shHAT1. Red, upregulated genes; Green, downregulated genes. b PANC-1 cells were infected with lentivirus expressing control, HAT1-specific shRNAs, pcDNA3.1, or HAT1 plasmid. After infecting $48 \mathrm{~h}$, cells were harvested to conduct RT-qPCR analysis. Statistical analyses were performed with one-way ANOVA followed by Tukey's multiple comparison's tests. All data were shown as mean values $\pm \mathrm{SD}(n=3) .{ }^{* *} P<0.01 ; * * * P<0.001$. $\mathbf{c}$, d The correlation analysis between HAT1 and PVT1 based on GEPIA web (c) and ENCORI Pan-Cancer Analysis Platform (d). e UCSC Genome Browser screenshots of the BRD4 ChIP-Sequence profiles at the PVT1 gene locus in C4-2 cells reported previously. f PANC-1, BxPC-3, and MIA-PaCa-2 cells were treated according to the protocol of ChIP experiment, and the resulting DNA sample was subjected to DNA agarose gel electrophoresis. All data were shown as mean values \pm SD $(n=3)$. ${ }^{* * * P<0.001 . ~ g ~ P A N C-1 ~ c e l l s ~ w e r e ~ i n f e c t e d ~ w i t h ~ l e n t i v i r u s ~ e x p r e s s i n g ~ c o n t r o l, ~ B R D 4-s p e c i f i c ~ s h R N A s, ~ a n d ~ B R D 4 ~}$ overexpressing plasmid. After infecting $48 \mathrm{~h}$, cells were harvested to conduct RT-qPCR analysis. All data were shown as mean values \pm SD $(n=$ 3). ${ }^{*} P<0.05 ; * * P<0.001$. $\mathbf{h}$ The correlation analysis between BRD4 and PVT1 based on GEPIA web. i PANC-1 cells were infected with lentivirus vectors expressing control, HAT1-specific shRNAs, BRD4-specific shRNAs, pcDNA3.1, or HAT1 plasmid. After $48 \mathrm{~h}$ infection, cells were harvested for RT-qPCR analysis. The data shown were the mean values \pm SD $(n=3)$. ns not significant; $* P<0.05 ; * * *<0.001$. j PANC-1 cells were infected with lentivirus vectors expressing control, HAT1-specific shRNAs, pcDNA3.1, or HAT1 plasmid. After $48 \mathrm{~h}$ infection, the cells were treated with or without JQ1 $(10 \mu \mathrm{M})$ for another $24 \mathrm{~h}$. Cells were harvested for RT-qPCR analysis. The data shown were the mean values \pm SD $(n=3)$. ns not significant; ${ }^{* *} P<0.001$. k PANC-1 cells were infected with pcDNA3.1 or HAT1 plasmid. After $48 \mathrm{~h}$ infection, the cells were treated with or without JQ1 $(10 \mu \mathrm{M})$ for another $24 \mathrm{~h}$. The cells were harvested for ChIP-qPCR analysis and DNA agarose gel electrophoresis. The data shown were the mean values \pm SD $(n=3)$. ns not significant; ${ }^{* * *} P<0.001$. I The schematic diagram of HAT1 regulating PVT1.

whereas HAT1 overexpression had the opposite effect (Fig. 3k). Moreover, restoring HAT1 expression into HAT1-deficient cells led to the decreased interaction between EZH2 and UBR4 (Fig. S5f). These data suggest that HAT1 competes with UBR4 for binding to the N-terminal domain of EZH2, thereby stabilizing EZH2 (Fig. 3I).

\section{HAT1 stabilizes EZH2 by competing with UBR4 for binding to EZH2}

We also found that UBR4 silencing profoundly increased the protein levels of EZH2 (Fig. 4a, b), as well as extended its half-life (Fig. 4c) and decreased its polyubiquitination levels (Fig. 4d, e), regardless of HAT1 silencing or overexpression. These data further confirmed that HAT1 stabilizes EZH2 by interfering with the binding of UBR4 to the N-terminus of EZH2.

\section{Preparation and characterization of chitosan (CS)- tripolyphosphate (TPP)-siHAT1}

CS-TPP-siRNA nanoparticles were prepared using an ionic gelation method and through crosslinking the negatively charged phosphate groups of TPP with the positively charged amino groups of CS [27]. At a 5:1 ratio, CS-TPP formed small, positively charged nanoparticles [28]. Hence, we prepared CS-TPP-siRNA nanoparticles by adding $250 \mu \mathrm{g}$ of siHAT 1 to a solution containing $1 \mathrm{mg}$ TPP. This mixture was added dropwise to a solution containing $5 \mathrm{mg}$ CS, yielding CS-TPP-siHAT1 nanoparticles (Fig. 5a). Transmission electron microscopy indicated that CS-TPP-siHAT1 particles had a spherical structure (Fig. 5b). The cellular uptake of CS-TPP-siRNA was also investigated by laser scanning confocal microscopy; strong fluorescent signals were observed in the cytoplasm (Fig. 5c). To assess the ability of CS-TPP-siHAT1 to suppress HAT1 expression, we incubated pancreatic cancer cells with different concentrations of CS-TPP-siHAT1 and for different times. A high concentration of CS-TPP-siHAT1 and prolonged incubation provided superior HAT1 downregulation, which also had a significant inhibitory effect on EZH2 and PVT1 (Fig. 5d, e and Fig. S5g).

\section{CS-TPP-siHAT1 augments the ability of gemcitabine to inhibit pancreatic cancer cell growth}

Next, we assessed the ability of CS-TPP-siHAT1 to enhance the cytotoxic effects of gemcitabine and found that the combination of CS-TPP-siHAT1 with gemcitabine was more potent in inhibiting cell proliferation than CS-TPP-siHAT1 or gemcitabine alone (Fig. $6 a, b$ and Fig. S6a, b). Additionally, Annexin-V/PI staining revealed that CS-TPP-siHAT1 augmented the proapoptotic effects of gemcitabine (Fig. 6c). In nude mice, CS-TPP-siHAT1 and gemcitabine were used to treat the nude, the detailed information was as follows (Fig. S6c). The combination of CS-TPP-siHAT1 with gemcitabine exhibited the most potent tumor-suppressive effects among all groups (Fig. $6 \mathrm{~d}-\mathrm{f}$ ). The delaminated tumors were also extracted proteins to analyze the expression of HAT1 (Fig. S6d). Additionally, tumors from mice treated with CS-TPP-siHAT1 combined with gemcitabine showed the highest caspase-3 levels and lowest Ki67 levels (Fig. 6g). Moreover, GR-PANC-1 was also used to further attest the synergistic effect, which showed that gemcitabine suppressed the growth of GR-PANC-1 and promoted the apoptosis of GR-PANC-1 after inhibiting HAT1 with CS-TPPsiHAT1 (Fig. S6e-g). These data suggested CS-TPP-siHAT1 and gemcitabine have a synergistic antitumor effect in pancreatic cancer.

\section{DISCUSSION}

Despite recent progress in cancer therapeutics, the prognosis of pancreatic cancer remains poor, primarily due to the development of gemcitabine resistance. The mechanisms underlying resistance to gemcitabine remain unclear and involve alterations in drug transporters, proteases, transcription factors, and drug metabolism enzymes $[29,30,7]$. These alterations can be internal to pancreatic cancer cells or induced by components of the tumor microenvironment [31].

HAT1 was first identified as a classical B type histone acetyltransferase mediating the acetylation of histone $\mathrm{H} 4$ $\mathrm{N}$-terminus [32]. As an epigenetic modifier, acetyl moieties can be found on lysine residues of cellular proteins (e.g., histones, transcription factors, nuclear receptors, and enzymes). In addition to regulating gene expression, protein acetylation plays a critical role in replication-dependent chromatin assembly and DNA damage repair [33, 34]. HAT1 mutations are frequent in tumors, leading to abnormal gene expression and promoting resistance to chemotherapeutic agents. For example, HAT1 was found to promote liver cancer cell proliferation and induce cisplatin resistance [17]. In melanoma, HAT1 was shown to catalyze histone $\mathrm{H} 4$ acetylation, thereby driving resistance to BET inhibitors [18]. In addition to HAT1 mutations, HAT1 overexpression is also frequent in multiple cancer types, including colon cancer [35], esophageal cancer [36], and lymphoma [37], exacerbating tumor malignancy. Hence, HAT1-mediated resistance to chemotherapy is an internal characteristic of tumor cells. We have previously shown that HAT1 was overexpressed in pancreatic cancer and that HAT1 silencing reduced the expression of PD-L1 on the surface of pancreatic cancer cells in a BRD4-dependent manner, improve the therapeutic efficacy of immune checkpoint blockade [38]. These findings suggest that HAT1 may represent an important therapeutic target in pancreatic cancer.

In this study, we found that HAT1 overexpression in pancreatic cancer cells promoted gemcitabine resistance and that 
a

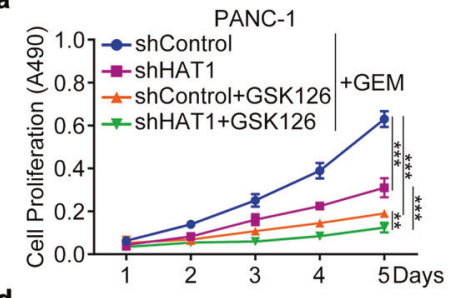

d

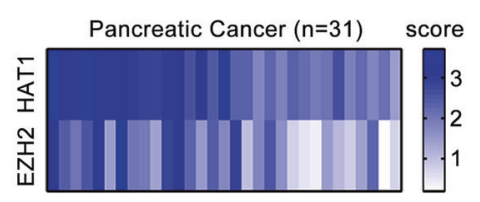

e

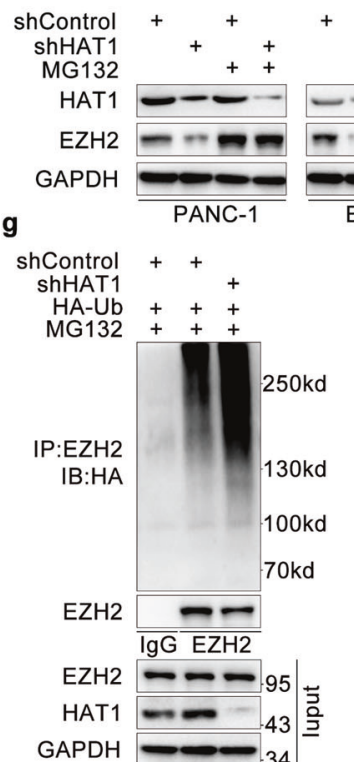

b

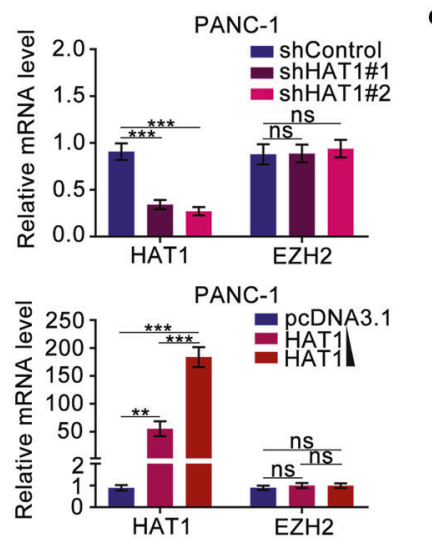

c

shControl +

EZH2

HAT1 - -

$\mathrm{GAPDH}-34$

PANC-1

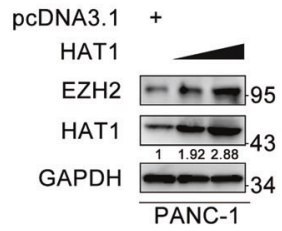

h

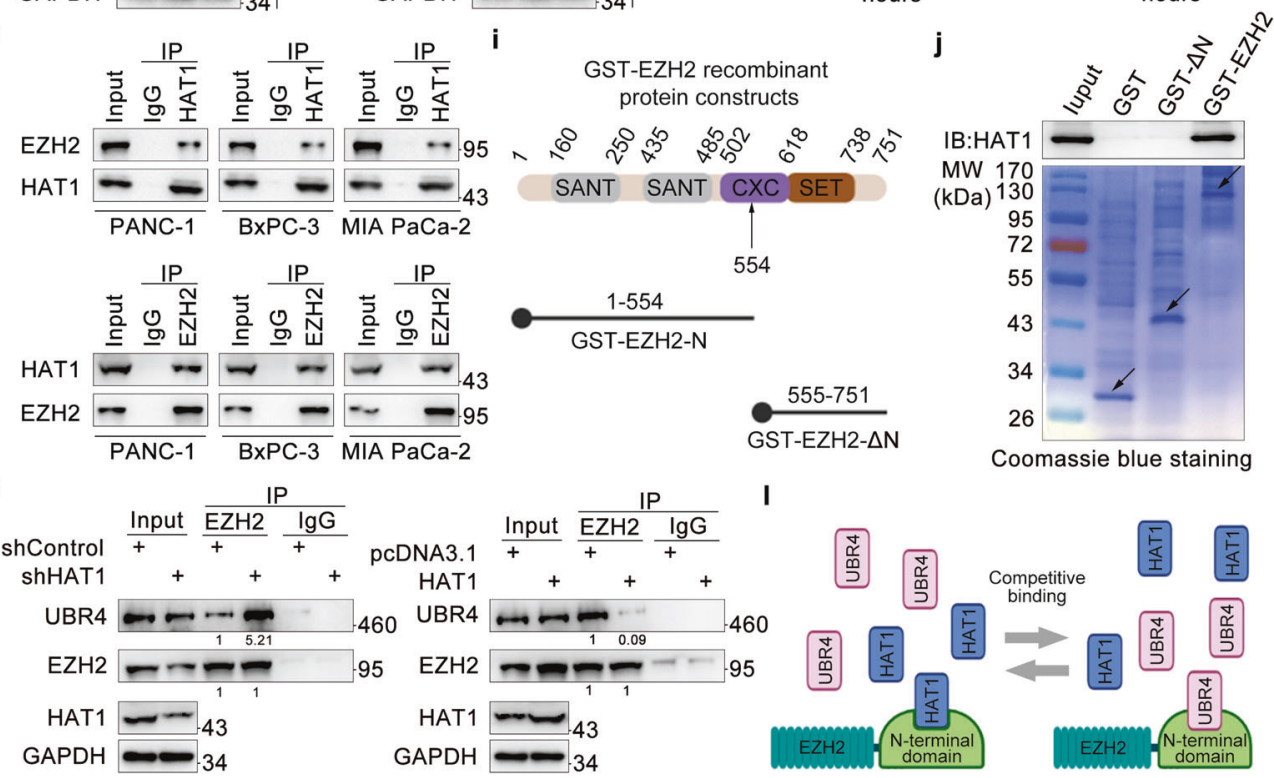

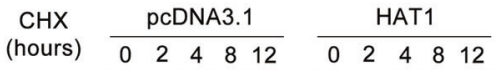

HAT1 -

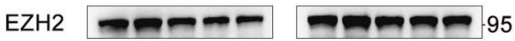

GAPDH 3

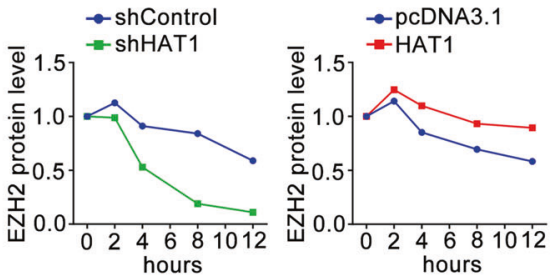

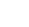


Fig. 3 HAT1 stabilizes EZH2 to promote gemcitabine resistance by competing with UBR4 for binding to the N-terminal domain of EZH2. a PANC-1 cells were infected with HAT1-specific shRNAs. After infecting $48 \mathrm{~h}$, cells were harvested and treated with GSK126 (10 $\mu \mathrm{M})$ and gemcitabine for 5 days to perform MTS assay. All data were shown as mean values \pm SD $(n=3) . * * P<0.01 ;{ }^{* * *} P<0.001$. b, c PANC- 1 cells were infected with lentivirus expressing control, HAT1-specific shRNAs and HAT1-overexpressing plasmid. After infecting $48 \mathrm{~h}$, cells were harvested for RT-qPCR analysis (b) and $72 \mathrm{~h}$ for western blotting analysis (c). Image $\mathrm{J}$ software was used to assess relative protein expression level. All data were shown as mean values \pm SD $(n=3)$. ns not significant; ${ }^{* *} P<0.01$; ${ }^{* *} P<0.001$. d HAT1 and EZH2 IHC score of tissue microarray of pancreatic cancer was plotted the heatmap. e PANC-1, BxPC-3, and MIA-PaCa-2 cells were infected with lentivirus expressing control or HAT1specific shRNAs. After infecting $48 \mathrm{~h}$, cells were treated with MG132 $(10 \mu \mathrm{M})$ for another $8 \mathrm{~h}$ and collected for western blotting analysis. $\mathrm{f}$ PANC1 cells were infected with indicated plasmids. After $72 \mathrm{~h}$ infection, cells were treated with Cycloheximide (CHX) and then collected for western blotting analysis at different timepoints. g PANC-1 were infected with lentivirus expressing control, HAT1-specific shRNAs, pCDNA3.1, or HAT1 plasmid. After $48 \mathrm{~h}$, PANC-1 cells were infected again with HA-Ub plasmid for $24 \mathrm{~h}$ and treated with MG132 $(10 \mu \mathrm{M})$ for another $8 \mathrm{~h}$ before harvesting cells. Western blotting analysis was conducted after co-immunoprecipitation. $\mathbf{h}$ Western blot analysis of endogenous HAT1 and EZH2 proteins reciprocally immunoprecipitated by anti-HAT1 and anti-EZH2 in PANC-1, BxPC-3, MIA-PaCa-2 cells. Immunoblots (IB) were representative of results from three independent experiments $(n=3)$. $\mathbf{i}$ Two GST-EZH2 recombinant proteins were constructed according to the structural representation: a whole-length GST-EZH2 and an N-terminus-lacking GST-EZH2 (GST- $\Delta$ N). $\mathbf{j}$ Western blot analysis of HAT1 proteins in PANC-1 pulled down by GST or GST-EZH2 recombinant proteins. Immunoblots were representative of results from three independent experiments $(n=3)$. The bottom panel shows Coomassie Blue staining of GST and GST-EZH2 recombinant protein input. Arrows, expected molecular weight. $\mathbf{k}$ The whole-cell lysate and Co-IP samples were analyzed by western blotting in PANC-1 cells after $48 \mathrm{~h}$ being infected with lentivirus expressing control shRNA or HAT1-specific shRNAs. UBR4 coimmunoprecipitated by EZH2 were quantified by Image J software and normalized to the quantified value of immunoprecipitated $\mathrm{EZH} 2$. The normalized values were further normalized to the value in cells infected with shControl. IB were representative of results from there independent experiments $(n=3)$. I The schematic diagram of HAT1 regulating $\mathrm{EZH} 2$.

cells, thereby inhibiting apoptosis and promoting resistance to 5-fluorouracil [41]. Additionally, PVT1 was found to upregulate the expression of numerous drug resistance-related molecules (e.g., MDR1 and MRP1) and inhibit apoptosis signaling, promoting cisplatin resistance in colorectal cancer. Notably, a previous genome-wide screening identified PVT1 as a critical regulator of gemcitabine sensitivity in pancreatic cancer [13]. Here, we confirmed the role of HAT1 in regulating PVT1 expression and identified BRD4 as a key player of the HAT1-mediated regulation of PVT1 expression.

Previous studies have shown that by forming a complex with EZH2, PVT1 promoted cell proliferation and inhibited apoptosis in liver cancer and thyroid cancer cells [23, 42]. The histone methyltransferase EZH2 suppresses gene expression by catalyzing the trimethylation of histone $\mathrm{H} 3$ lysine 27 (H3K27me3) [43, 44]. $\mathrm{EZH} 2$ has also been implicated in multidrug resistance in gastric cancer and ovarian cancer $[45,46]$. Importantly, EZH2 has been shown to promote drug resistance by suppressing $\mathrm{p} 27^{\mathrm{Kip} 1}$ expression in pancreatic cancer cells [12]. Our study was consistent with previous research results, which showed $\mathrm{EZH} 2$ regulated the gemcitabine sensitivity and PVT1 could bind to EZH2. More importantly, we found that HAT1 enhanced the expression of EZH2 in pancreatic cancer cells in this study. We also found that HAT1 regulated $\mathrm{EZH} 2$ expression at the post-transcriptional rather than transcriptional level. Moreover, we found that HAT1 interacted with $\mathrm{EZH} 2$, increasing the stability of the latter. Jalan-Sakrikar $\mathrm{N}$ et al. found that the E3 ubiquitin ligase UBR4 could bind to the $\mathrm{N}$-terminus of $\mathrm{EZH} 2$, promoting $\mathrm{EZH} 2$ ubiquitination and subsequent degradation [26]. Consistent with these results, we found that HAT1 could bind to the N-terminus of EZH2, interfering with the ability of UBR4 to interact with EZH2 (Fig. 3I).

This study suggests that HAT1 regulates the sensitivity of pancreatic cancer to gemcitabine by regulating PVT1/EZH2 complex, highlighting the importance of HAT1 in the development of gemcitabine resistance in pancreatic cancer. Although we provide strong evidence that HAT1 inhibition may suppress tumor growth, reverse drug resistance, and improve the prognosis of pancreatic cancer, there are currently no HAT1 small molecule inhibitors. High-throughput screening approaches have led to the identification of potential drug candidates; however, most of these compounds exhibited moderate efficacy and specificity [47]. Therefore, future studies are urgently needed to develop specific and potent HAT1 inhibitors. Nanoparticles carrying siRNA have emerged as promising alternatives of small molecule inhibitors [48-50]. Here, we used a CS-TPP carrier to deliver siHAT1 and suppress HAT1 expression in pancreatic cancer cells. CS-TPPsiHAT1 nanoparticles have proved effective in inhibiting HAT1 expression and augmenting the antitumor effects of gemcitabine in pancreatic cancer. The effectiveness and safety of CS-TPPsiHAT1 nanoparticles required further investigation in a clinical setting.

\section{CONCLUSIONS}

Our data strongly support that HAT1 upregulates PVT1 and promotes gemcitabine resistance in pancreatic cancer by enhancing BRD4 binding to the PVT1 promoter. We also show that HAT1 prevents $\mathrm{EZH} 2$ degradation by preventing UBR4 binding to the $\mathrm{N}$-terminal domain of EZH2. Our findings suggest that HAT1induced gemcitabine resistance in pancreatic cancer may be mediated by the PVT1/EZH2 complex. Finally, we show that CSPTT-siHAT1 nanoparticles suppress HAT1 expression and augment the antitumor effects of gemcitabine in pancreatic cancer cells. Collectively, the findings presented here suggest that HAT1 may be a valuable therapeutic target in pancreatic cancer.

\section{MATERIALS AND METHODS}

\section{Cell lines and cell culture}

Pancreatic cancer cell lines (PANC-1, BxPC-3, and MIA-PaCa-2) were purchased from the Chinese Academy of Science Cell Bank. Cell lines were cultured in RPMI 1640 medium (Gibco, USA) containing 10\% fetal bovine serum (FBS; Gibco, USA), $100 \mathrm{IU} / \mathrm{mL}$ penicillin, and $100 \mu \mathrm{g} / \mathrm{mL}$ streptomycin (Gibco, USA). Plasmocin (InvivoGen) was routinely added to the medium to eliminate mycoplasma. All cell lines were maintained at $37{ }^{\circ} \mathrm{C}$ in a $5 \% \mathrm{CO}_{2}$ incubator.

\section{Cell transfection}

Cells were transfected with different plasmids using Lipofectamine 2000 Reagent (Thermo Fisher Scientific). Small hairpin RNAs (shRNAs) targeting HAT1 (shHAT1, shBRD4, shUBR4, shPVT1, and shEZH2) and overexpression plasmid (pcDNA3.1 backbone vector, HAT1, BRD4, and PVT1) were obtained from Shanghai GeneChem Co., Ltd. Opti-MEM medium (Gibco, USA) was used to prepare the transfection mixtures. Six hours after transfection, the Opti-MEM medium was replaced with 10\% FBScontaining RPMI 1640 medium. The sequences of shHAT1 and shBRD4 are provided in Table 1.

\section{Lentiviral and the construction of stable cell lines}

Lentiviral particles carrying gene-specific shRNAs and negative control shRNA (hU6-MCS-CBh-gcGFP-IRES-puromycin) were used to infect 
a

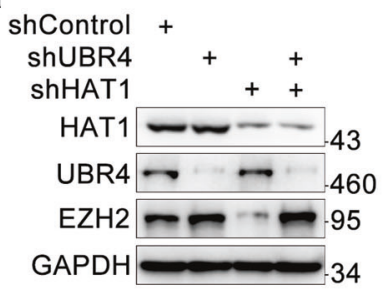

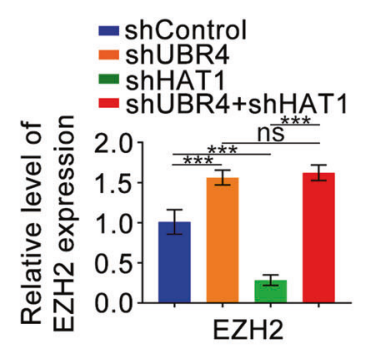

c

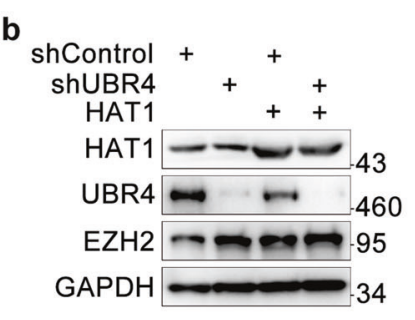

d

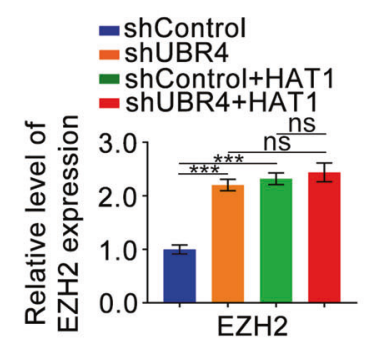

shControl ++

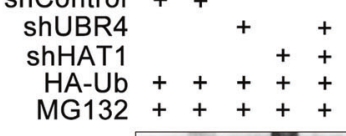

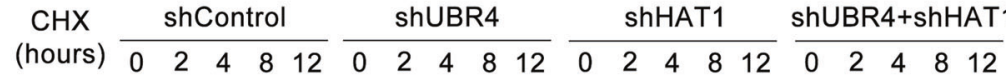
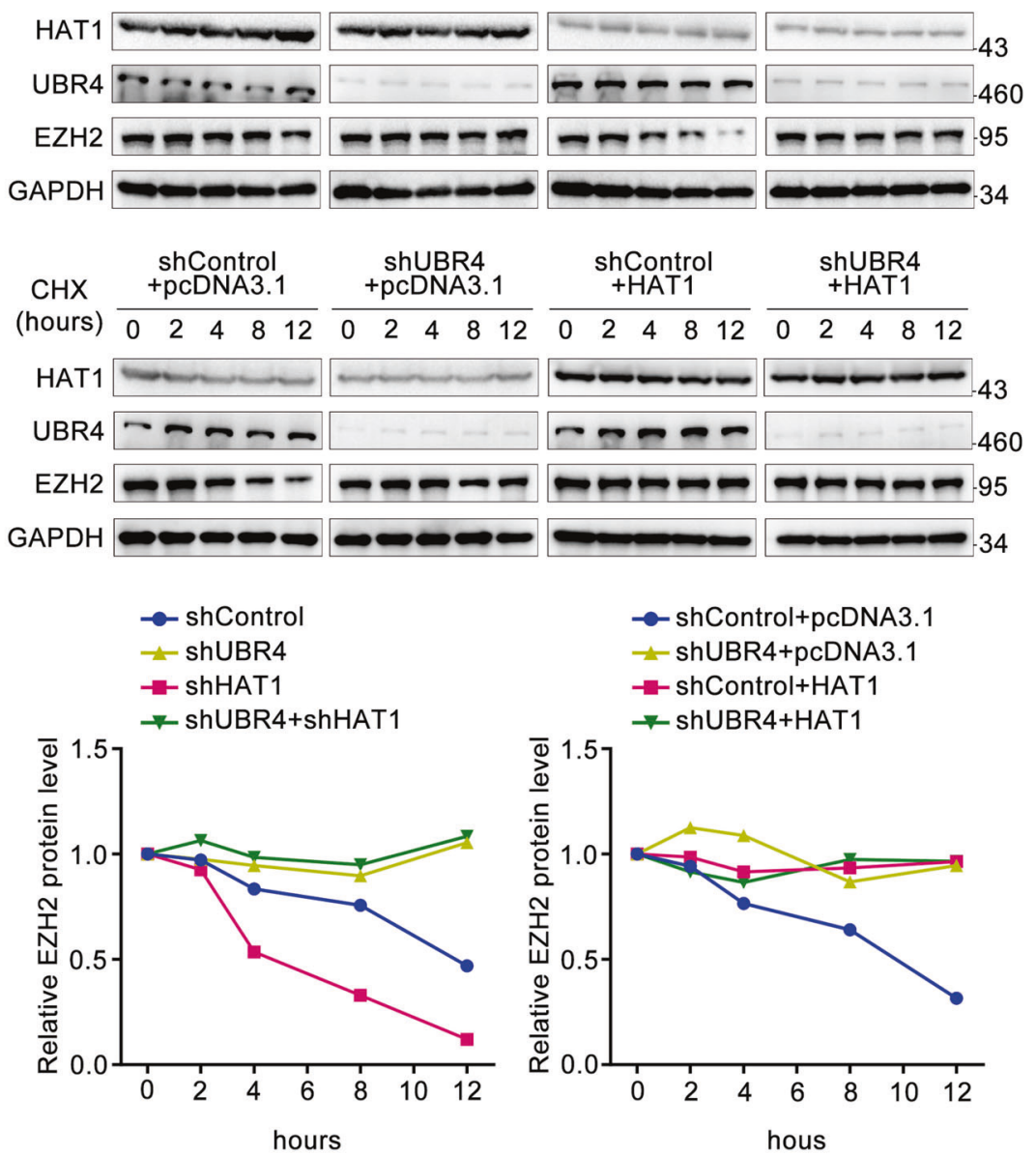

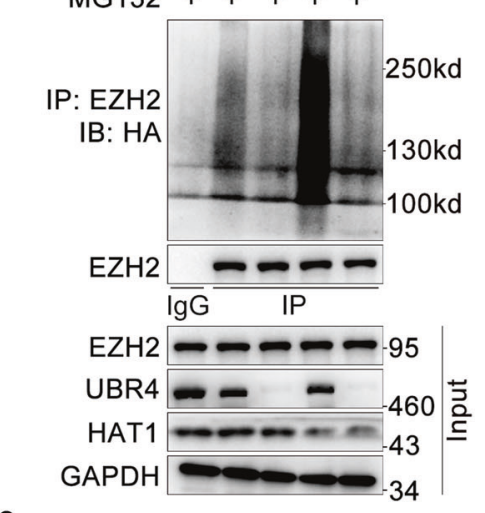

e

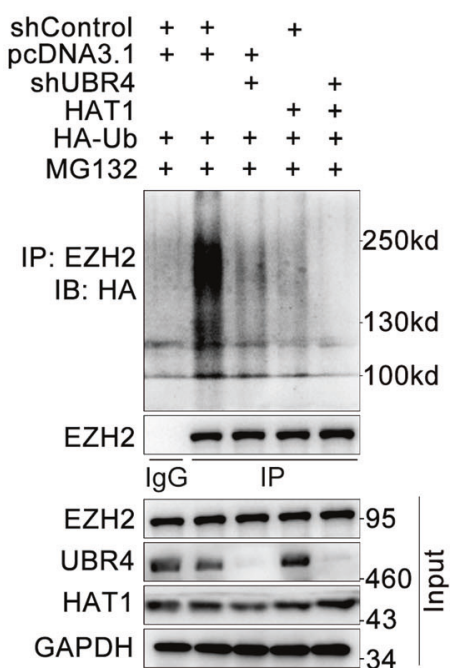

Fig. 4 HAT1 stabilizes EZH2 by competing with UBR4 for binding to EZH2. a, b PANC-1 cells were infected with lentivirus expressing control, HAT1-specific shRNAs, UBR4-specific shRNAs, and HAT1-overexpressing plasmid. After infecting $72 \mathrm{~h}$, cells were harvested for western blotting analysis $(n=3)$. c PANC-1 cells were infected with indicated plasmids. After $72 \mathrm{~h}$ infection, cells were treated with Cycloheximide $(\mathrm{CHX})$ and then collected for western blotting analysis at different timepoints. d, e PANC-1 cells were infected with indicated plasmids. After $48 \mathrm{~h}$, PANC-1 cells were infected again with HA-Ub plasmid for $24 \mathrm{~h}$ and treated with MG132 $(10 \mu \mathrm{M})$ for another $8 \mathrm{~h}$ before harvesting cells. Western Blotting analysis was conducted after co-immunoprecipitation.

pancreatic cancer cells. Briefly, pancreatic cancer cells were seeded in sixwell plates $\left(2 \times 10^{5}\right.$ cells per well). The day after, $1 \mathrm{~mL}$ of DMEM medium containing the viral particles and $40 \mu \mathrm{L}$ of Hitrans $\mathrm{G}$ reagent was added to each well. After $16 \mathrm{~h}$, the viral solution was replaced with complete growth medium. Puromycin was used to select infected cells.

\section{Antibodies and chemicals}

In this study, the following antibodies were used: anti-HAT1 (11432-1-AP, Proteintech; 1:1000 dilution), anti-EZH2 (21800-1-AP, Proteitech; 1:1000 dilution), anti-UBR4 (ab86738, Abcam; 1:2000 dilution), anti-caspase-3 (19677-1-AP, Proteintech; 1:2000 dilution), anti-GAPDH (10494-1-AP, Proteintech; 1:2000 dilution), anti-HA (51064-2-AP, Proteintech; 1:1000 dilution), anti-Flag (20543-1-AP, Proteintech; 1:1000 dilution), anti-H4 (16047-1-AP, Proteintech; 1:1000 dilution), anti-H4ac (AB 2687872, 1:1000 dilution). The following chemicals were used: gemcitabine (T0251, Topscience; $50 \mathrm{nM}$ in vitro and $10 \mathrm{mg} / \mathrm{kg}$ in vivo), JQ1 (Cat. No. S7110, Selleck; working concentration $10 \mu \mathrm{M}$ ), MG132 (HY-13259, MedChemExpress; working concentration $10 \mu \mathrm{M}$ ), and GSK126 (HY-13470, MedChemExpress; working concentration $10 \mu \mathrm{M}$ ).

\section{Immunohistochemistry (IHC)}

Pancreatic cancer tissue microarrays (HPan-Ade060CD-01) were purchased from Shanghai Outdo Biotech Co, Ltd and obtained the ethical committee approval. Tissues were stained with anti-HAT1 (1:2000 dilution) and anti- 


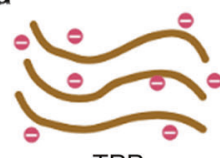

TPP
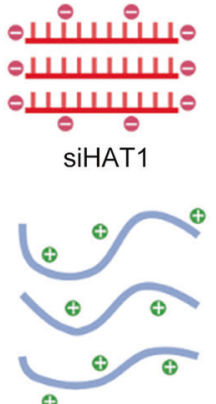

CS
CS molecular structure
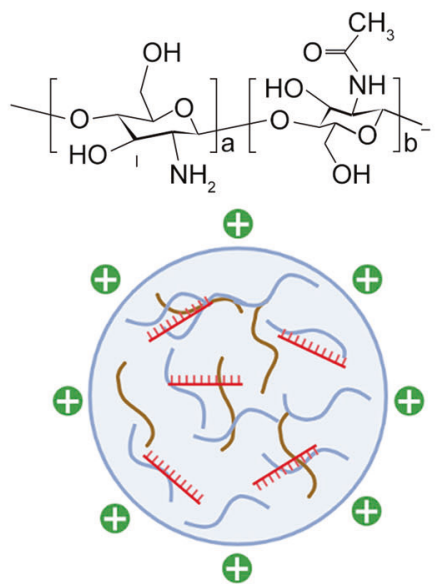

CS-TPP-SiHAT1

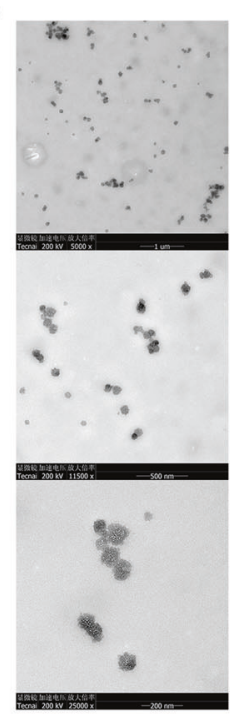

BxPC-3

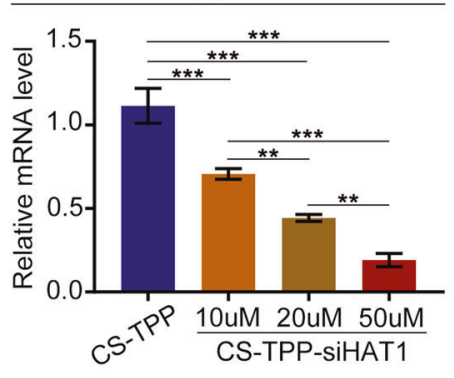

CS-TPP +

CS-TPP-siHAT1
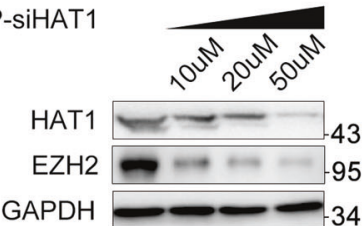

BxPC-3

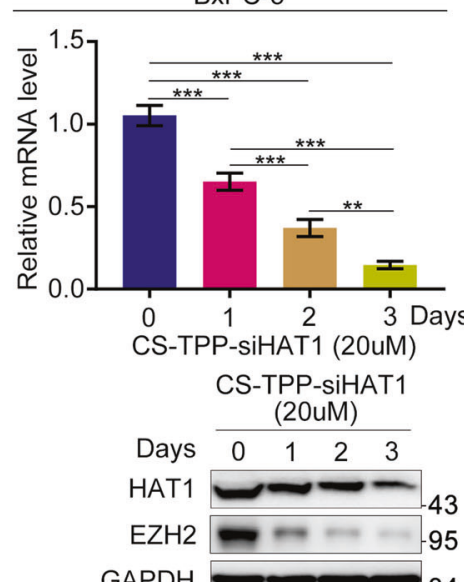

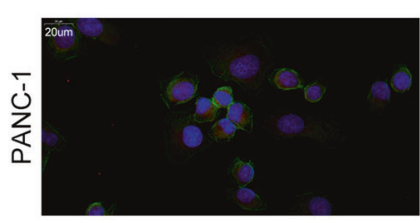
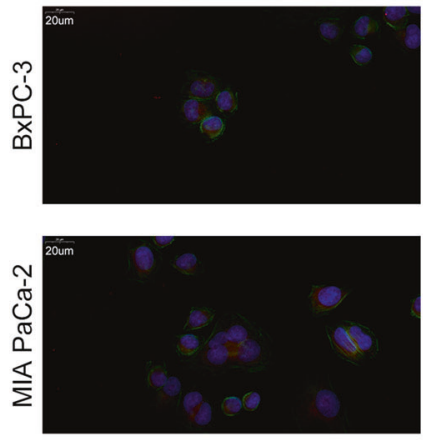

MIA PaCa-2

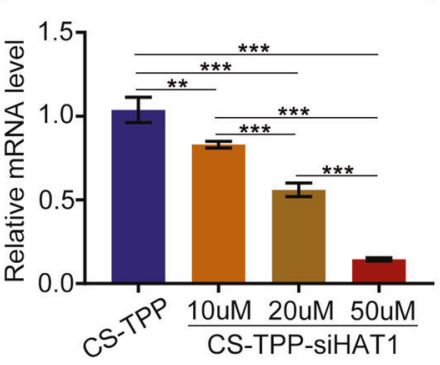

CS-TPP +

CS-TPP-siHAT1
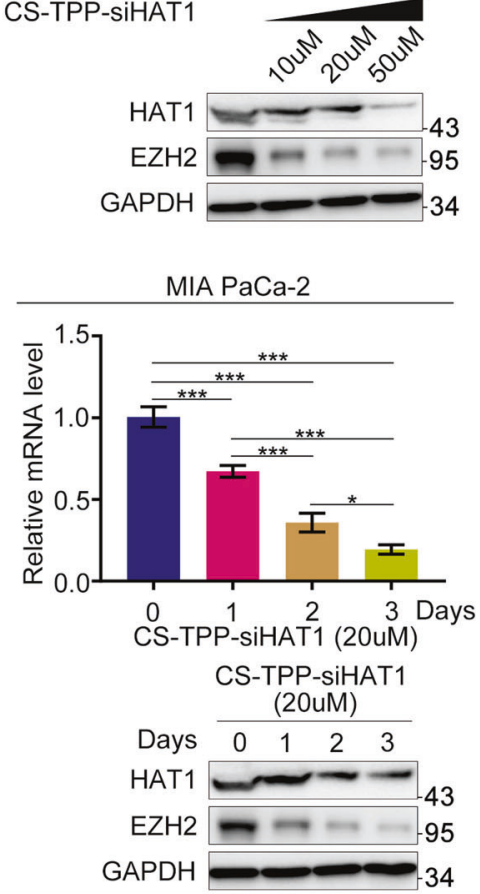

EZH2 (1:1000 dilution). The IHC score was determined independently by two experienced pathologists blinded to the study groups. The staining intensity was scored as follows: 1 , weak staining at $\times 100$ magnification and little or no staining at $\times 40$ magnification; 2 , moderate staining at $\times 40$ magnification; 3 , strong staining at $\times 40$ magnification.

\section{Cell proliferation and colony formation assay}

The MTS (3-(4,5-dimethylthiazol-2-yl)-5-(3-carboxymethoxyphenyl)-2-(4sulfophenyl)-2H-tetrazolium) reagent (ab197010, Abcam, USA) was used to assess cell proliferation in vitro. Cells were seeded in 96-well plates (2000 cells in $100 \mu \mathrm{l}$ DMEM per well) and cultured for 3 days. Subsequently, 
Fig. 5 Preparation and characterization of chitosan (CS)-tripolyphosphate (TPP)-siHAT1. a The scheme for CS-TPP-siHAT1 preparation. 250 ug siHAT1 was added into $1 \mathrm{mg}$ tripolyphosphate (TPP) solution. Then, the above mixture solution was added into $5 \mathrm{mg}$ chitosan (CS) solution dropwise and stirring slowly for $30 \mathrm{~min}$ to prepare the CS-TPP-siHAT1.TPP tripolyphosphate, CS chitosan. $\mathbf{b}$ Different magnifications TEM images of CS-TPP-siHAT1. c The cellular uptake confocal images of CS-TPP-siRNA in PANC-1, BxPC-3, and MIA-PaCa cells. d PANC-1, BxPC3 , and MIA-PaCa cells were treated with different CS-TPP-siHAT1 concentration $(10 \mu \mathrm{M}, 20 \mu \mathrm{M}, 50 \mu \mathrm{M})$ for 3 days. The cells were harvested to extract total protein and RNA respectively, and then RT-qPCR and western blotting were performed to analyzed HAT1, PVT1, and EZH2 expression level. All data were shown as mean values \pm SD $(n=3) . * P<0.05 ; * * P<0.01 ; * * P<0.001$. e PANC-1, BxPC-3, and MIA-PaCa cells were treated with $20 \mu \mathrm{M}$ CS-TPP-siHAT1 for different time ( 0 days, 1 days, 2 days, 3 days). The cells were harvested to extract total protein and RNA, respectively, and then RT-qPCR and western blotting were performed to analyzed HAT1, PVT1, and EZH2 expression level. All data were shown as mean values \pm SD $(n=3)$. ${ }^{*} P<0.05 ; * * P<0.01 ; * * * P<0.001$.

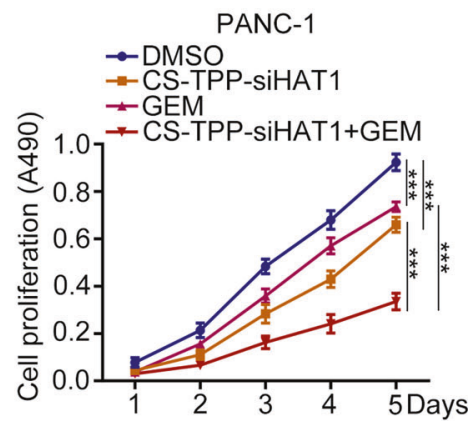

C

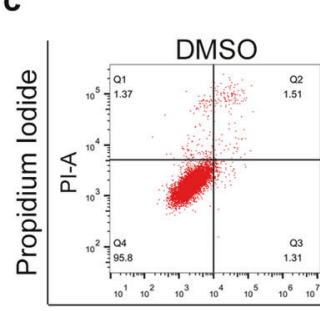

d

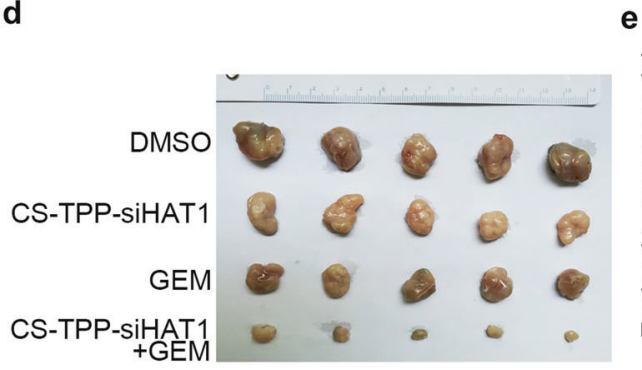

g

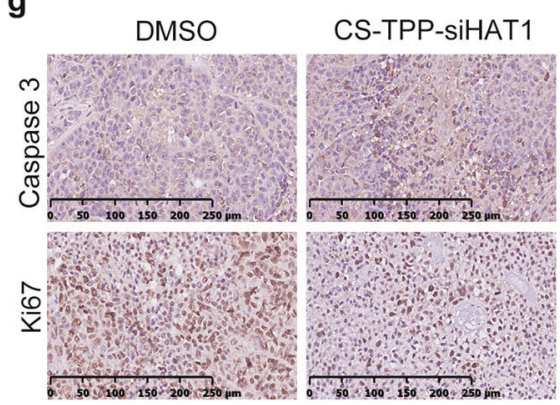

b
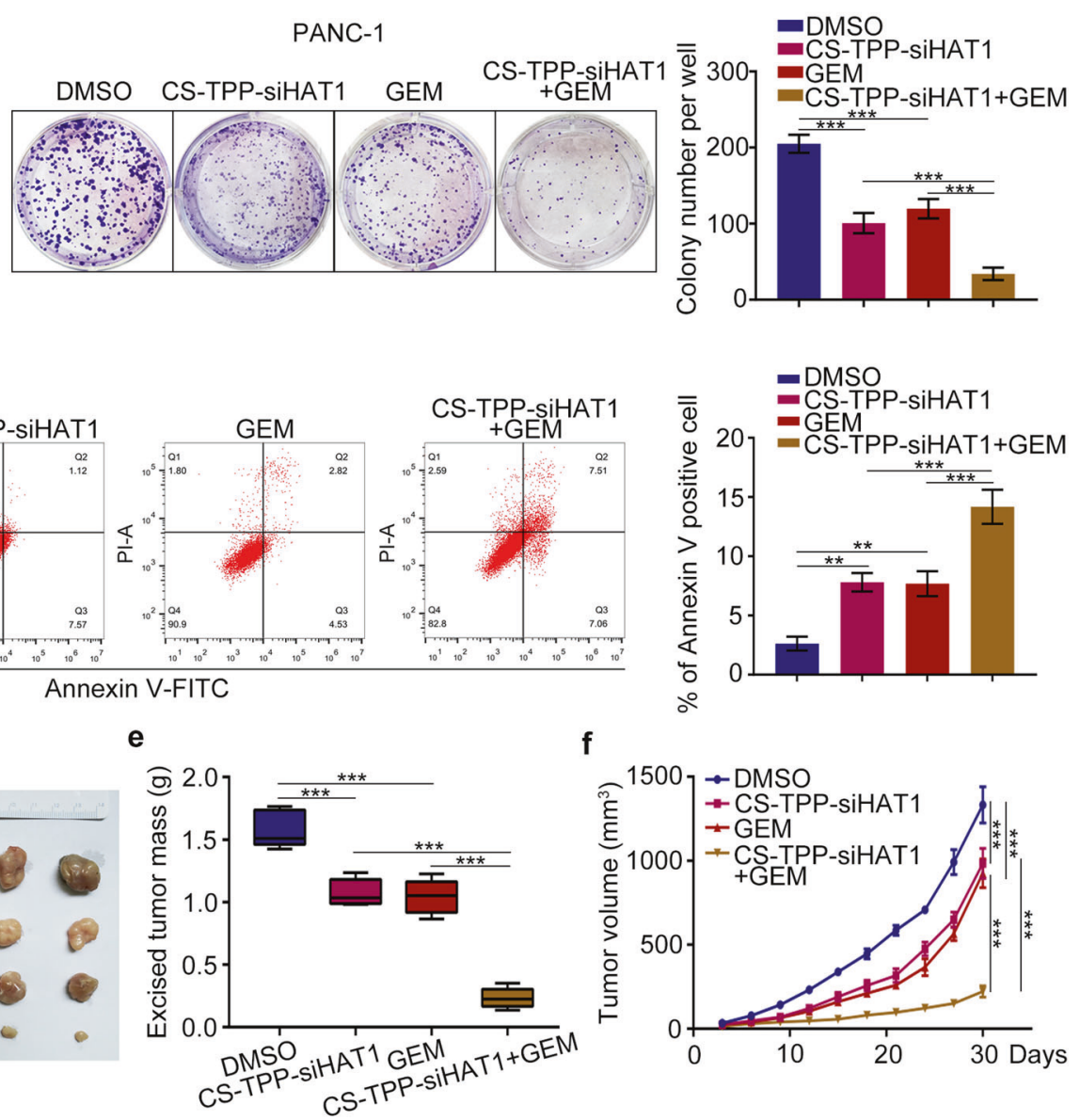

f

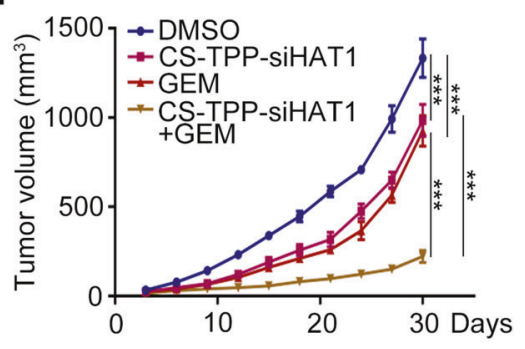

Fig. 6 CS-TPP-siHAT1 augments the ability of gemcitabine to inhibit pancreatic cancer cell growth. a-c PANC-1 cells were treated with CSTPP-siHAT1 alone, gemcitabine alone or the combination for MTS assay (a), clone formation assay (b), and Annexin-V/Propidium lodide assay (c). All data were shown as mean values \pm SD $(n=3)$. ${ }^{*} P<0.01 ; * * P<0.001$. d-f PANC-1 cells were subcutanousely injected into the nude mice. These mice were injected intraperitoneally with CS-TPP-siHAT1 $(50 \mu \mathrm{M})$ alone, gemcitabine $(10 \mathrm{mg} / \mathrm{kg})$ alone or the combination every 3 days. The tumor volume was measured every 3 days and all tumors were harvested for photograph and weight after 30 days $(n=5)$. $* * * P<$ 0.001 . g All tumors were conducted caspase-3 and Ki67 analysis by IHC staining. All data were shown as means values \pm SD $(n=5)$. ${ }^{* * *} P<$ 0.001 . 
Table 1. The sequences of gene-specific shRNAs.

ShHAT1\#1

ShHAT1\#2

ShHAT1\#3

ShBRD4\#1

ShBRD4\#2

ShUBR4\#1

ShUBR4\#2

ShPVT1\#1

ShPVT1\#2

ShEZH2\#1

ShEZH2\#2
5' - CCGGGCTACATGACAGTCTATAATTCTCGAGAATTATAGACTGTCATGTAGCTTTTTG-3'

5'- CCGGCCGTGTTGAATATGCATCTAACTCGAGTTAGATGCATATTCAACACGGTTTTTG-3' 5'-CCGGGCAAGGATTCAATGAAGATATCTCGAGATATCTTCATTGAATCCTTGCTTTTTG-3' 5'-CCGGCCTGGAGATGACATAGTCTTACTCGAGTAAGACTATGTCATCTCCAGGTTTTTG-3' 5'-CCGGCAGTGACAGTTCGACTGATGACTCGAGTCATCAGTCGAACTGTCACTGTTTTTG-3' 5'-CCGGGCCGCTGATGAGGGATATAAACTCGAGTTTATATCCCTCATCAGCGGCTTTTTTG-3' 5'-CCGGCCACCATCAAAGACTTACATTCTCGAGAATGTAAGTCTTTGATGGTGGTTTTTTG-3' 5'-ACCGGACTTGAGAACTGTCCTTACCGAAGTAAGGACAGTTCTCAAGTCC-3' 5'-CACCGCTTCTCCTGTTGCTGCTAGTCGAAACTAGCAGCAACAGGAGAAGC-3' 5'-CCGGCCCAACATAGATGGACCAAATCTCGAGATTTGGTCCATCTATGTTGGGTTTTTG-3' 5'-CCGGTATGATGGTTAACGGTGATCACTCGAGTGATCACCGTTAACCATCATATTTTTG-3'

Table 2. The sequences of RT-qPCR primers.

\begin{tabular}{ll} 
Gene & Forward primer $\mathbf{( 5}^{\prime} \mathbf{- 3}^{\prime} \mathbf{)}$ \\
\hline HAT1 & GGATGGAGCTACGCTCTTTG \\
\hline PVT1 & ATAGATCCTGCCCTGTTTGC \\
\hline EZH2 & CCCTGACCTCTGTCTTACTTGTGGA \\
\hline BRD4 & ACCTCCAACCCTAACAAGCC \\
\hline GAPDH & ACCCACTCCTCCACCTTTGAC \\
\hline
\end{tabular}

Reverse primer $\left(5^{\prime}-3^{\prime}\right)$ GGATGGATCTTCCGCTGTAA CATTTCCTGCTGCCGTTTTC ACGTCAGATGGTGCCAGCAATA TTTCCATAGTGTCTTGAGCACC TGTTGCTGTAGCCAAATTCGTT

Table 3. The sequence of ChIP-qPCR primers.

$\begin{array}{llll}\text { ChIP target } & \text { Gene } & \text { Forward primer }\left(\mathbf{5}^{\prime}-\mathbf{3}^{\prime}\right) & \text { Reverse primer }\left(5^{\prime}-\mathbf{3}^{\prime}\right) \\ \text { BRD4 } & \text { PVT1 } & \text { AGGGATGCGCTGTGAGTAGT } & \text { TTCTGACTGCAGAGGGGTCT }\end{array}$

$20 \mu \mathrm{l}$ MTS reagent was added to each well, and cells were incubated for $4 \mathrm{~h}$ in the dark. Optical absorbance at $490 \mathrm{~nm}$ was measured on a microplate reader after adding $200 \mu \mathrm{L}$ DMSO.

Cells were seeded in six-well plates (500 cells/well) for colony formation assays. The cell growth medium was replaced every 3 days. After 2 weeks, cells were fixed with $4 \%$ paraformaldehyde $(30 \mathrm{~min})$ and stained with crystal violet (20 $\mathrm{min})$.

\section{The preparation of gemcitabine-resistant PANC-1}

Gemcitabine was dissolved in dimethyl sulfoxide (DMSO) at a concentration of $1 \mathrm{mM}$. Then, gemcitabine solution was diluted to a working concentration of $50 \mathrm{nM}$ with RPMI 1640 medium containing 10\% FBS. The pancreatic cancer cell line PANC-1 was cultured with the above configured medium for 2 weeks in $5 \% \mathrm{CO} 2$ at $37^{\circ} \mathrm{C}$. Then the cells were cultured for another 2 weeks without gemcitabine. The above process was repeated three times. The selected cells could resist gemcitabine and the cells were named GR-PANC-1.

\begin{abstract}
Western blotting
Cells were lysed by sonication in RIPA lysis buffer (P0013B, Beyotime) containing $1 \%$ protease inhibitor. The cell lysate was centrifuged at $12,000 \mathrm{rpm}$ and $4^{\circ} \mathrm{C}$, and the supernatant was collected and boiled for $10 \mathrm{~min}$ at $95^{\circ} \mathrm{C}$. The protein concentration was measured using Lowry method with protein concentration determination kit (Solarbio, PC0030). Subsequently, proteins were resolved by SDS-PAGE and transferred onto PVDF membranes (Pierce Biotechnology, USA). Membranes were blocked with $5 \%$ skim milk for $1 \mathrm{~h}$ at room temperature and incubated with primary antibodies overnight at $4{ }^{\circ} \mathrm{C}$. The next day, the membranes were washed three times and incubated with the respective secondary antibody for $1 \mathrm{~h}$ at room temperature. Signal was developed using $\mathrm{ECL}$ chemiluminescence reagent and X-ray films.
\end{abstract}

Quantitative real-time PCR (qRT-PCR)

Total RNA was extracted using TRIzol reagent (15596026, Invitrogen, USA). PrimeScript ${ }^{\text {TM }}$ RT kit (Takara, Japan) was used to synthesize CDNA, which was subsequently amplified using TB Green ${ }^{\mathrm{TM}}$ Fast qPCR Mix (Takara, Japan). Relative gene expression was calculated using the $2^{-\Delta C q}$ method after normalizing to GAPDH levels. The sequences of the qRT-PCR primers are shown in Table 2.

\section{Co-immunoprecipitation (Co-IP) and chromatin immunoprecipitation (ChIP)}

Cells were lysed in western/IP lysis buffer for $30 \mathrm{~min}$ at $4^{\circ} \mathrm{C}$, and the cell lysate was centrifuged for $10 \mathrm{~min}$ at $12,000 \mathrm{rpm}$ and $4^{\circ} \mathrm{C}$. The supernatant was incubated with protein A/G agarose beads (Thermo Fisher Scientific, USA) overnight at $4{ }^{\circ} \mathrm{C}$ with gentle agitation. The protein $A / G$ agarose beads were collected and washed seven times with western/IP lysis buffer. Subsequently, proteins were resolved by SDS-PAGE.

According to the manufacturer's protocols, chromatin Immunoprecipitation (ChIP) assay was carried out through utilizing the Chromatin Extraction Kit (Abcam, ab117152, USA) and ChIP Kit Magnetic-One Step (Abcam, ab156907, USA) [38, 51]. BRD4 antibody (Cell Signaling Technology, 13440, working concentration 1:50) was used to perform ChIP assay for precipitating the promoter of PVT1. The precipitated DNA fragments were further amplified via quantitative real-time PCR with a PCR kit (Takara Bio Inc., Japan) based on the manufacturer's instructions [38, 52]. The sequences of the primers used for ChIP-qPCR are provided in Table 3.

\section{RNA immunoprecipitation (RIP) assay}

According to the manufacturer's protocols, RNA immunoprecipitation (RIP) assay was performed through using Magna RIP RNA-Binding Protein Immunoprecipitation Kit (Millipore, MA, USA). The supernatant of cell lysate was extracted and then mixed up with treated beads to incubate for $6 \mathrm{~h}$. Then the RIP wash buffer was used to wash the beads for seven times. At last, qRT-PCR analysis was conducted to analyze the purified RNA.

RNA pull-down assay

mMESSAGE mMACHINE T7 Kit (Ambion, USA) and RNeasy Mini Kit (Qiagen, Valencia, CA) were used to transcribe PVT1 and the antisense RNA in vitro, 
and Pierce RNA $3^{\prime}$ End Desthiobiotinylation Kit (Thermo Scientific, USA) was utilized to conduct biotin labeling. One milligram total protein extracts were added to $50 \mathrm{pmol}$ of biotin-labeled PVT1 for incubating $1 \mathrm{~h}$, then mixed up with $60 \mu \mathrm{L}$ of Streptavidin Beads (Invitrogen) for another $1 \mathrm{~h}$. Finally, Western blotting analysis was used to detect the proteins.

\section{Flow cytometry}

Annexin-V-FITC/PI kit (AntGene, China) was used to assess apoptosis. Cells treated with different agents were harvested and washed with phosphate buffer saline (PBS). Cells were incubated with annexin-V-FITC and PI according to the manufacturer's instructions. Flow cytometry was performed on BD FACSCelesta (BD Biosciences, USA), and the data were analyzed using FlowJo

\section{Confocal imaging}

Pancreatic cancer cells were seeded into slide chambers, and after overnight incubation, cells were incubated with chitosan (CS)-tripolyphosphate (TPP)-siHAT1 $(10 \mu \mathrm{g} / \mathrm{mL})$ for $4 \mathrm{~h}$. Cells were washed with PBS, fixed in $4 \%$ paraformaldehyde, and permeabilized with $0.2 \%$ Triton X-100 for $5 \mathrm{~min}$. Subsequently, cells were treated with $100 \mathrm{nM}$ FITC-phalloidin for $1 \mathrm{~h}$ at $37^{\circ} \mathrm{C}$ and in the dark. Cell nuclei were counterstained with Hoechst 33342 for $5 \mathrm{~min}$. Stained cells were observed under a laser confocal microscope.

\section{Subcutaneous xenotransplantation tumor model}

Nude mice (18-20 g, 5 weeks old, male) were purchased from Vitalriver (Beijing, China) and maintained under specific pathogen-free (SPF) conditions. Mice were randomly divided into groups (five mice per group) and subcutaneously injected with $3 \times 10^{6}$ PANC-1 cells. The volume was measured every 3 days (volume $=\left(L \times W^{2}\right) / 2$ ). The treatment protocols with drugs were as follows: When the tumors reached to $50 \mathrm{~mm}^{3}$, experimental groups were injected intraperitoneally gemcitabine $(10 \mathrm{mg} / \mathrm{kg})$ or CS-TPPsiHAT1 $(50 \mu \mathrm{M})$ with every 3 days, control groups were injected intraperitoneally equal DMSO or CS-TPP. After 30 days, mice were sacrificed, and tumors were excised and weighed. All animal experimental procedures were approved by the Ethics Committee of Tongji Medical College, Huazhong University of Science and Technology.

\section{Glutathione S-transferase (GST) pull-down assay}

GST fusion proteins were immobilized on glutathione-sepharose beads (GE Healthcare Life Sciences), and the beads were incubated with cell lysis buffer $(20 \mathrm{mmol} / \mathrm{L} \mathrm{Tris}-\mathrm{HCl}$ [pH 7.5], $150 \mathrm{mmol} / \mathrm{L} \mathrm{NaCl}, 0.1 \%$ Nonidet P40, $1 \mathrm{mmol} / \mathrm{L}$ dithiothreitol [DTT], $10 \%$ glycerol, $1 \mathrm{mmol} / \mathrm{L}$ EDTA, $2.5 \mathrm{mmol} / \mathrm{L} \mathrm{MgCl}_{2}$, and $1 \mu \mathrm{g} / \mathrm{mL}$ leupeptin) for $4 \mathrm{~h}$. After incubation and washing, the beads were resuspended in protein loading buffer and subjected to SDS-PAGE.

\section{Statistical analysis}

All experiments were replicated for three times. All data were analyzed using two-tailed with GraphPad Prism 7.0. Student's $t$-test, one-way analysis of variance (ANOVA), and two-way ANOVA was used to evaluate statistical significance (similar variance). $P$ values $<0.05$ were considered statistically significant. All values were expressed as means \pm SD.

\section{DATA AVAILABILITY}

All data generated or analyzed during this study are included in this published article and its supplementary information files.

\section{REFERENCES}

1. Kong B, Michalski CW, Erkan M, Friess H, Kleeff J. From tissue turnover to the cell of origin for pancreatic cancer. Nat Rev Gastroenterol Hepatol. 2011;8:467-72.

2. Stanger BZ, Dor Y. Dissecting the cellular origins of pancreatic cancer. Cell Cycle. 2006;5:43-46.

3. Tascilar M, Skinner HG, Rosty C, Sohn T, Wilentz RE, Offerhaus GJ, et al. The SMAD4 protein and prognosis of pancreatic ductal adenocarcinoma. Clin Cancer Res. 2001;7:4115-21.

4. Yeo CJ, Cameron JL. Prognostic factors in ductal pancreatic cancer. Langenbeck's Arch Surg. 1998;383:129-33.

5. Ferlay J, Soerjomataram I, Dikshit R, Eser S, Mathers C, Rebelo M, et al. Cancer incidence and mortality worldwide: sources, methods and major patterns in GLOBOCAN 2012. Int J Cancer. 2015;136:E359-E386.
6. Hidalgo M, Cascinu S, Kleeff J, Labianca R, Löhr JM, Neoptolemos J, et al. Addressing the challenges of pancreatic cancer: future directions for improving outcomes. Pancreatology. 2015;15:8-18.

7. Binenbaum Y, Na'ara S, Gil Z. Gemcitabine resistance in pancreatic ductal adenocarcinoma. Drug Resist Updat. 2015;23:55-68.

8. Minami K, Shinsato $Y$, Yamamoto $M$, Takahashi $H$, Zhang $S$, Nishizawa $Y$, et al. Ribonucleotide reductase is an effective target to overcome gemcitabine resistance in gemcitabine-resistant pancreatic cancer cells with dual resistant factors. J Pharmacol Sci. 2015;127:319-25.

9. Kim MP, Gallick GE. Gemcitabine resistance in pancreatic cancer: picking the key players. Clin Cancer Res. 2008;14:1284-5.

10. de Sousa Cavalcante L, Monteiro G. Gemcitabine: metabolism and molecular mechanisms of action, sensitivity and chemoresistance in pancreatic cancer. Eur J Pharmacol. 2014;741:8-16.

11. Zhang Y-J, Wen C-L, Qin Y-X, Tang X-M, Shi M-M, Shen B-Y, et al. Establishment of a human primary pancreatic cancer mouse model to examine and investigate gemcitabine resistance. Oncol Rep. 2017;38:3335-46.

12. Ougolkov AV, Bilim VN, Billadeau DD. Regulation of pancreatic tumor cell proliferation and chemoresistance by the histone methyltransferase enhancer of zeste homologue 2. Clin Cancer Res. 2008;14:6790-6.

13. You L, Chang D, Du H-Z, Zhao Y-P. Genome-wide screen identifies PVT1 as a regulator of Gemcitabine sensitivity in human pancreatic cancer cells. Biochem Biophys Res Commun. 2011;407:1-6.

14. Yoshida K, Toden S, Ravindranathan P, Han H, Goel A. Curcumin sensitizes pancreatic cancer cells to gemcitabine by attenuating PRC2 subunit EZH2, and the IncRNA PVT1 expression. Carcinogenesis. 2017;38:1036-46.

15. Miyamoto N, Izumi H, Noguchi T, Nakajima Y, Ohmiya $Y$, Shiota M, et al. Tip60 is regulated by circadian transcription factor clock and is involved in cisplatin resistance. J Biol Chem. 2008;283:18218-26.

16. Bugide S, Parajuli KR, Chava S, Pattanayak R, Manna DLD, Shrestha D, et al. Loss of HAT1 expression confers BRAFV600E inhibitor resistance to melanoma cells by activating MAPK signaling via IGF1R. Oncogenesis. 2020;9:44.

17. Jin X, Tian S, Li P. Histone acetyltransferase 1 promotes cell proliferation and induces cisplatin resistance in hepatocellular carcinoma. Oncol Res. 2017;25:939-46.

18. Mustafi S, Camarena V, Volmar C-H, Huff TC, Sant DW, Brothers SP, et al. Vitamin C sensitizes melanoma to BET inhibitors. Cancer Res. 2018;78:572-83.

19. Zhang $P$, Wang D, Zhao $Y$, Ren $S$, Gao K, Ye Z, et al. Intrinsic BET inhibitor resistance in SPOP-mutated prostate cancer is mediated by BET protein stabilization and AKT-mTORC1 activation. Nat Med. 2017;23:1055-62.

20. Zhou L, Mudianto T, Ma X, Riley R, Uppaluri R. Targeting EZH2 enhances antigen presentation, antitumor immunity, and circumvents anti-PD-1 resistance in head and neck cancer. Clin Cancer Res. 2020;26:290-300.

21. Adelaiye-Ogala R, Budka J, Damayanti NP, Arrington J, Ferris M, Hsu CC, et al. EZH2 modifies sunitinib resistance in renal cell carcinoma by kinome reprogramming. Cancer Res. 2017;77:6651-66.

22. Xu Y, Lian Y, Zhang Y, Huang S, Zuo Q, Yang N, et al. The long non-coding RNA PVT1 represses ANGPTL4 transcription through binding with EZH2 in trophoblast cell. J Cell Mol Med. 2018;22:1272-82.

23. Guo J, Hao C, Wang C, Li L. Long noncoding RNA PVT1 modulates hepatocellular carcinoma cell proliferation and apoptosis by recruiting EZH2. Cancer Cell Int. 2018;18:98.

24. Han Z, Xing $X$, Hu M, Zhang $Y$, Liu $P$, Chai J. Structural basis of EZH2 recognition by EED. Structure. 2007;15:1306-15.

25. Jin X, Yang C, Fan P, Xiao J, Zhang W, Zhan S, et al. CDK5/FBW7-dependent ubiquitination and degradation of EZH2 inhibits pancreatic cancer cell migration and invasion. J Biol Chem. 2017;292:6269-80.

26. Jalan-Sakrikar N, De Assuncao TM, Shi G, Aseem SO, Chi C, Shah VH, et al. Proteasomal degradation of enhancer of zeste homologue 2 in cholangiocytes promotes biliary fibrosis. Hepatology. 2019;70:1674-89.

27. Katas H, Alpar HO. Development and characterisation of chitosan nanoparticles for siRNA delivery. J Control Release. 2006;115:216-25.

28. Dhandapani RK, Gurusamy D, Howell JL, Palli SR. Development of CS-TPP-dsRNA nanoparticles to enhance RNAi efficiency in the yellow fever mosquito, Aedes aegypti. Sci Rep. 2019;9:8775.

29. Zeng S, Pöttler M, Lan B, Grützmann R, Pilarsky C, Yang H. Chemoresistance in pancreatic cancer. Int J Mol Sci. 2019;20:4504.

30. Shukla SK, Purohit V, Mehla K, Gunda V, Chaika NV, Vernucci E, et al. MUC1 and HIF-1alpha signaling crosstalk induces anabolic glucose metabolism to impart gemcitabine resistance to pancreatic cancer. Cancer Cell. 2017;32:71-87.

31. Bergman AM, Pinedo HM, Peters GJ. Determinants of resistance to 2 ', 2 '-difluorodeoxycytidine (gemcitabine). Drug Resist Updat. 2002;5:19-33.

32. Yang X, Li L, Liang J, Shi L, Yang J, Yi X, et al. Histone acetyltransferase 1 promotes homologous recombination in DNA repair by facilitating histone turnover. J Biol Chem. 2013;288:18271-82. 
33. Audia JE, Campbell RM. Histone modifications and cancer. Cold Spring Harb Perspect Biol. 2016;8:a019521.

34. Sadler AJ, Suliman BA, Yu L, Yuan X, Wang D, Irving AT, et al. The acetyltransferase HAT1 moderates the NF-KB response by regulating the transcription factor PLZF. Nat Commun. 2015;6:6795.

35. Seiden-Long IM, Brown KR, Shih W, Wigle DA, Radulovich N, Jurisica I, et al. Transcriptional targets of hepatocyte growth factor signaling and Ki-ras oncogene activation in colorectal cancer. Oncogene. 2006;25:91-102.

36. Xue L, Hou J, Wang Q, Yao L, Xu S, Ge D. RNAi screening identifies HAT1 as a potential drug target in esophageal squamous cell carcinoma. Int J Clin Exp Pathol. 2014;7:3898-907.

37. Min SK, Koh YH, Park Y, Kim HJ, Seo J, Park H-R, et al. Expression of HAT1 and HDAC1, 2, 3 in diffuse large B-cell lymphomas, peripheral T-cell lymphomas, and NK/T-cell lymphomas. Korean J Pathol. 2012;46:142-50.

38. Fan P, Zhao J, Meng Z, Wu H, Wang B, Wu H, et al. Overexpressed histone acetyltransferase 1 regulates cancer immunity by increasing programmed death-ligand 1 expression in pancreatic cancer. J Exp Clin Cancer Res. 2019;38:47.

39. Graham M, Adams JM. Chromosome 8 breakpoint far 3' of the c-myc oncogene in a Burkitt's lymphoma $2 ; 8$ variant translocation is equivalent to the murine pvt-1 locus. EMBO J. 1986;5:2845-51.

40. Shtivelman E, Henglein B, Groitl P, Lipp M, Bishop JM. Identification of a human transcription unit affected by the variant chromosomal translocations 2;8 and 8;22 of Burkitt lymphoma. Proc Natl Acad Sci USA. 1989;86:3257-60

41. Du $P, H u$ CE, Qin $Y$, Zhao J, Patel $R, F u ~ Y$, et al. LncRNA PVT1 mediates antiapoptosis and 5-fluorouracil resistance via increasing $\mathrm{Bcl} 2$ expression in gastric cancer. J Oncol. 2019;2019:9325407.

42. Zhou Q, Chen J, Feng J, Wang J. Long noncoding RNA PVT1 modulates thyroid cancer cell proliferation by recruiting $\mathrm{EZH} 2$ and regulating thyroid-stimulating hormone receptor (TSHR). Tumour Biol. 2016;37:3105-13.

43. Cao R, Wang L, Wang H, Xia L, Erdjument-Bromage $H$, Tempst $P$, et al. Role of histone H3 lysine 27 methylation in Polycomb-group silencing. Science. 2002;298:1039-43.

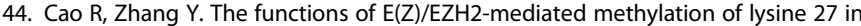
histone H3. Curr Opin Genet Dev. 2004;14:155-64.

45. Wang $\mathrm{C}$, Li X, Zhang J, Ge Z, Chen $\mathrm{H}$, Hu J. EZH2 contributes to 5-FU resistance in gastric cancer by epigenetically suppressing FBXO32 expression. Onco Targets Ther. 2018;11:7853-64.

46. Abbosh PH, Montgomery JS, Starkey JA, Novotny M, Zuhowski EG, Egorin MJ, et al. Dominant-negative histone $\mathrm{H} 3$ lysine 27 mutant derepresses silenced tumor suppressor genes and reverses the drug-resistant phenotype in cancer cells. Cancer Res. 2006;66:5582-91.

47. Marmorstein R, Zhou M-M. Writers and readers of histone acetylation: structure, mechanism, and inhibition. Cold Spring Harb Perspect Biol. 2014;6: a018762.

48. Sun Q, Wang X, Cui C, Li J, Wang Y. Doxorubicin and anti-VEGF siRNA co-delivery via nano-graphene oxide for enhanced cancer therapy in vitro and in vivo. Int J Nanomed. 2018;13:3713-28.

49. Zhu X, Xu Y, Solis LM, Tao W, Wang L, Behrens $C$, et al. Long-circulating siRNA nanoparticles for validating Prohibitin1-targeted non-small cell lung cancer treatment. Proc Natl Acad Sci USA. 2015;112:7779-84.

50. Duo $Y$, Yang $M$, Du Z, Feng $C$, Xing C, Wu Y, et al. CX-5461-loaded nucleolustargeting nanoplatform for cancer therapy through induction of pro-death autophagy. Acta Biomater. 2018;79:317-30.

51. Fan P, Wang B, Meng Z, Zhao J, Jin X. PES1 is transcriptionally regulated by BRD4 and promotes cell proliferation and glycolysis in hepatocellular carcinoma. Int J Biochem cell Biol. 2018;104:1-8.
52. Jin $X$, Yan $Y$, Wang D, Ding D, Ma T, Ye Z, et al. DUB3 promotes BET inhibitor resistance and cancer progression by deubiquitinating BRD4. Mol Cell. 2018;71:592-605.e594

\section{AUTHOR CONTRIBUTIONS}

$\mathrm{XJ}$ and HW conceived and designed this project, and reviewed and wrote the final manuscript; YS, JS, and DR designed and conducted all experiments, analyzed the data; $Y Z$ generated the immune-histochemistry data and labelled the image.

\section{FUNDING INFORMATION}

This work was supported by grants from the National Natural Science Foundation of China (Grant No. 82073321 (XJ)) and Science Foundation of Ministry of Education of China (Grant No. 2172019kfyRCPY069 (XJ)).

\section{COMPETING INTERESTS}

The authors declare no competing interests.

\section{ETHICS APPROVAL AND CONSENT TO PARTICIPATE}

All animal experimental procedures were approved by the Ethics Committee of Tongji Medical College, Huazhong University of Science and Technology. This study did not involve human participants, human data, or human tissue.

\section{ADDITIONAL INFORMATION}

Supplementary information The online version contains supplementary material available at https://doi.org/10.1038/s41419-021-04118-4.

Correspondence and requests for materials should be addressed to Jian Shen Heshui Wu or Xin Jin.

Reprints and permission information is available at http://www.nature.com/ reprints

Publisher's note Springer Nature remains neutral with regard to jurisdictional claims in published maps and institutional affiliations.

\begin{abstract}
Open Access This article is licensed under a Creative Common Attribution 4.0 International License, which permits use, sharing, adaptation, distribution and reproduction in any medium or format, as long as you give appropriate credit to the original author(s) and the source, provide a link to the Creative Commons license, and indicate if changes were made. The images or other third party material in this article are included in the article's Creative Commons license, unless indicated otherwise in a credit line to the material. If material is not included in the article's Creative Commons license and your intended use is not permitted by statutory regulation or exceeds the permitted use, you will need to obtain permission directly from the copyright holder. To view a copy of this license, visit http://creativecommons. org/licenses/by/4.0/.
\end{abstract}

(c) The Author(s) 2021 